\title{
Asymmetric Type-la supernova origin of W49B as revealed from spatially resolved X-ray spectroscopic study
}

\author{
Ping Zhou ${ }^{1,2}$ and Jacco Vink ${ }^{1,3}$ \\ ${ }^{1}$ Anton Pannekoek Institute, University of Amsterdam, PO Box 94249, 1090 GE Amsterdam, The Netherlands \\ e-mail: p.zhou, j.vink@uva.nl \\ ${ }^{2}$ School of Astronomy and Space Science, Nanjing University, Nanjing 210023, PR China \\ ${ }^{3}$ GRAPPA, University of Amsterdam, PO Box 94249, 1090 GE Amsterdam, The Netherlands
}

Received 17 July 2017 / Accepted 23 March 2018

\begin{abstract}
The origin of the asymmetric supernova remnant (SNR) W49B has been a matter of debate: is it produced by a rare jet-driven corecollapse (CC) supernova, or by a normal supernova that is strongly shaped by its dense environment? Aiming to uncover the explosion mechanism and origin of the asymmetric, centrally filled X-ray morphology of W49B, we have performed spatially resolved X-ray spectroscopy and a search for potential point sources. We report new candidate point sources inside W49B. The Chandra X-ray spectra from W49B are well-characterized by two-temperature gas components $(\sim 0.27 \mathrm{keV}+0.6-2.2 \mathrm{keV})$. The hot component gas shows a large temperature gradient from the northeast to the southwest and is over-ionized in most regions with recombination timescales of $1-10 \times 10^{11} \mathrm{~cm}^{-3} \mathrm{~s}$. The Fe element shows strong lateral distribution in the SNR east, while the distribution of $\mathrm{Si}, \mathrm{S}, \mathrm{Ar}, \mathrm{Ca}$ is relatively smooth and nearly axially symmetric. Asymmetric Type-Ia explosion of a Chandrasekhar-mass white dwarf (WD) wellexplains the abundance ratios and metal distribution of $\mathrm{W} 49 \mathrm{~B}$, whereas a jet-driven explosion and normal CC models fail to describe the abundance ratios and large masses of iron-group elements. A model based on a multi-spot ignition of the WD can explain the observed high $M_{\mathrm{Mn}} / M_{\mathrm{Cr}}$ value (0.8-2.2). The bar-like morphology is mainly due to a density enhancement in the center, given the good spatial correlation between gas density and X-ray brightness. The recombination ages and the Sedov age consistently suggest a revised SNR age of 5-6 kyr. This study suggests that despite the presence of candidate point sources projected within the boundary of this SNR, W49B is likely a Type-Ia SNR, which suggests that Type-Ia supernovae can also result in mixed-morphology SNRs.
\end{abstract}

Key words. ISM: individual objects: W49B - ISM: supernova remnants - nuclear reactions, nucleosynthesis, abundances - white dwarfs

\section{Introduction}

The study of supernova remnants (SNRs) provides information about both the supernova explosions themselves, and about the environments in which the supernova explosions took place. The environment often carries important information about the supernova progenitor itself, such as whether it formed in a star-forming region, and whether the progenitor shaped its own environment with a stellar wind. In particular, massive stars are known to create large wind-blown bubbles of several tens of parsecs in size (Weaver et al. 1977; Chevalier 1999).

The morphology and spectra of SNRs are determined by the combined effects of both the intrinsic explosion properties and the ambient medium in which they involve. Unfortunately, however, it is sometimes difficult to disentangle the effects of explosion properties and the environment in which they occurred. There are properties that can be firmly attributed to the explosion properties, but also properties that may be attributed to either the explosion characteristics or to the environment. For example, for young SNRs it is clear that the abundance pattern provides clear signatures of the type of explosion, with Type-Ia supernovae producing more iron-group elements (IGEs), whereas core-collapse (CC) SNRs are more abundant in oxygen, neon, and magnesium (Hughes et al. 1995; Vink 2012).

Mixed-morphology SNRs are a special class of SNRs characterized by bright thermal X-ray emission from their center, and a shell-type morphology in the radio. Initially, it was noted that mixed-morphology SNRs show thermal emission in the interior originating from low-abundant hot gas (Rho \& Petre 1998; Jones et al. 1998). W49B was included in the list, but appeared to have enhanced abundances. However, increasingly more of the originally mixed-morphology SNRs appeared to also have enhanced abundances in their interiors (Lazendic \& Slane 2006). It is clear that W49B, together with some other metalrich cases such as Sgr A East (Sakano et al. 2004; Park et al. 2005) stand out. In most reviews of mixed-morphology SNRs, W49B is listed as a mixed-morphology SNR (Lazendic \& Slane 2006; Vink 2012; Zhang et al. 2015; Dubner \& Giacani 2015), and the definition of mixed-morphology SNR is in those cases based solely on different radio and X-ray morphology, and the fact that the X-ray emission is thermal in nature. It is thought that mixed-morphology SNRs evolve in denser environments, and since massive stars are associated with molecular cloud environments, it is usually assumed that these SNRs are remnants of CC supernovae. Indeed, some of the mixed-morphology SNRs have associated young pulsars, proving that these SNRs are indeed CC SNRs (e.g., W44, IC 443, Wolszczan et al. 1991; Olbert et al. 2001).

Thermonuclear (or Type-Ia) supernova progenitors are carbon-oxygen white dwarfs (WDs), which take a longer time to evolve ( $>40 \mathrm{Myr}$ ), and, moreover, only explode if they accrete sufficient matter from a companion star (the single-degenerate 
scenario; Whelan \& Iben 1973), or merge with a companion carbon-oxygen WD (the double-degenerate scenario; Webbink 1984). By the time they explode, their ambient medium does not necessarily contain any information anymore about their progenitors. The exact origin of Type-Ia supernovae is still a source of debate (see reviews Branch et al. 1995; Hillebrandt \& Niemeyer 2000; Livio 2000; Wang \& Han 2012; Maoz et al. 2014, and references therein), but also the manner in which the WDs explode is uncertain, with models involving deflagration (Nomoto et al. 1984), competing with so-called delayed detonation (DDT) models (Khokhlov 1991). In general, Type-Ia SNRs are often to be found in less dense regions of the Galaxy. For example, SN 1006 is found high above the Galactic plane ( $b=14.6^{\circ}$, corresponding to $\sim 560 \mathrm{pc}$ at a distance of $2.18 \pm$ $0.08 \mathrm{kpc}$, Winkler et al. 2003). The less disturbed media in which they are often found may account for the generally more symmetric morphology, as compared to CC SNRs (Lopez et al. 2011). On the other hand, the more symmetric morphologies of TypeIa SNRs may also be caused by intrinsically more symmetric explosions.

The idea that Type-Ia progenitors do not shape the supernova environments has recently been challenged. For example, it is clear that Kepler's SNR (Vink 2016, for a review), a TypeIa SNR (Kinugasa \& Tsunemi 1999; Reynolds et al. 1994), is evolving inside a bow-shock-shaped high-density region caused by the wind from a progenitor system (Chiotellis et al. 2012). In contrast, the likely Type-Ia SNR RCW 86 (see Gvaramadze et al. 2017, for a recent paper suggesting a CC origin) seems to evolve inside the low-density environment created by a powerful lowdensity wind (Williams et al. 2011; Broersen et al. 2014). Type-Ia SNR Tycho is suggested to be overrunning a slowly expanding molecular bubble created by its progenitor's outflow (Zhou et al. 2016). The middle-aged SNR G299.2-2.9 is a Type-Ia SNR showing asymmetries in the ejecta distribution due to an asymmetric explosion and/or a nonuniform surrounding medium (Post et al. 2014; Park et al. 2007). Moreover, some Type-Ia supernovae may explode with intrinsic asymmetries (Röpke et al. 2007; Maeda et al. 2010b), which has been used to interpret the spectral evolution diversity observed in Type-Ia supernovae (Maeda et al. 2010a).

Many of the above-mentioned issues of relating SNRs and their environments to the explosion types, come together in the peculiar SNR W49B, which is suggested at a distance of 8$11.3 \mathrm{kpc}$ (Radhakrishnan et al. 1972; Brogan \& Troland 2001; Chen et al. 2014; Zhu et al. 2014). The X-ray emission from W49B is dominated by emission from the center, which was initially attributed to the presence of a pulsar wind nebula (Pye et al. 1984), but was not much later discredited by the fact that the X-ray spectra obtained by the EXOSAT satellite displayed bright $\mathrm{Fe}-\mathrm{K}$ lines (Smith et al. 1985). Due to the centrally enhanced X-ray morphology, it was listed in the first catalog of mixed-morphology SNRs, but with a metal-rich interior (Rho \& Petre 1998). However, the brightness of the Fe-K lines and detailed spectroscopy with ASCA (Hwang et al. 2000) suggested that W49B is relatively young (1000-4000 yr) compared to most mixed-morphology SNRs, although it does share some characteristics with Sgr A East, a metal-rich, mixed-morphology SNR (Maeda et al. 2002). A property that W49B shares with many other mixed-morphology SNRs is that the plasma appears over-ionized (Kawasaki et al. 2005; Yamaguchi et al. 2009; Miceli et al. 2010), rather than under-ionized, as in most SNRs. Like many mixed-morphology SNRs, W49B is a GeV gammaray source, but it is also a TeV gamma-ray source, which is more rare for this class of SNRs (H. E. S. S. Collaboration 2018).
Although the X-ray spectrum of W49B shows the SNR to be very iron-rich, it is usually assumed that it is a CC SNR, like most mixed-morphology SNRs, albeit a peculiar one. Hwang et al. (2000) expressed some doubts, suggesting that neither a $\mathrm{CC}$ origin, nor a Type-Ia origin could explain the measured abundances. The brightness of the $\mathrm{Fe}-\mathrm{K}$ lines, but also the peculiar, jet-like morphology of the ejecta, has been interpreted as evidence that $\mathrm{W} 49 \mathrm{~B}$ is the result of a hypernova explosion (Keohane et al. 2007). On the other hand, Miceli et al. (2006) compared the observed abundances with yields for hypernova and supernova nucleosynthesis and found better agreement for the abundances of W49B with models with a normal explosion energy $\left(10^{51} \mathrm{erg}\right)$. More recently, Lopez et al. (2013b), assuming W49B to be a CC SNR, presented evidence that the supernova produced a black hole rather than a neutron star (NS). The reason is that they did not find evidence for a cooling NS, similar to the X-ray point source in Cas A (Tananbaum 1999).

The study presented here was prompted by the many peculiarities of W49B. Most notably, we were puzzled by the fact that black holes are thought to be the end products of the most massive stars (>25 $M_{\odot}$, e.g., Heger et al. 2003), but W49B seems to be evolving in a cavity of only $\sim 5 \mathrm{pc}$ radius (Keohane et al. 2007). In contrast, a progenitor more massive than $25 M_{\odot}$ is expected to create a cavity with a radius of at least $20 \mathrm{pc}$ (Chen et al. 2013).

With our study we therefore tried to investigate, a) whether a cooling NS may, after all, be present, given that W49B provides a spatially non-uniform X-ray background that could hide a point source, and that the interstellar absorption is relatively high $\left(N_{\mathrm{H}}>10^{22} \mathrm{~cm}^{-2}\right.$; and b) whether W49B is indeed a CC SNR or even a jet-driven CC SNR, as often assumed.

To answer these questions we reanalyzed the archival Chandra data, using a state-of-the-art adaptive binning method for spatially resolved X-ray spectroscopy, and we made a new search for X-ray point sources. We indeed found a few point sources, but our overall conclusion is that the X-ray spectra fit better with a Type-Ia origin for $\mathrm{W} 49 \mathrm{~B}$, and in particular that the abundance pattern best fits the multi-point ignition DDT models of Seitenzahl et al. (2013b).

\section{Data and analysis}

\subsection{Data}

W49B was observed with Chandra in three epochs in 2000 (obs. ID: 117; PI: Stephen Holt) and 2011 (obs. IDs: 13440 and 13441; PI: Laura Lopez), with exposures of 54, 158, and $60 \mathrm{ks}$, respectively. We retrieve three sets of Chandra data, which covered the SNR with the backside-illuminated S3 chip in the faint mode. We use CIAO software (vers. 4.9 and CALDB vers. 4.7 .7$)^{1}$ to reduce the data, extract spectra, and detect the point-like sources. Xspec (vers. $12.9 .0 \mathrm{u})^{2}$ is used for spectral analysis.

\subsection{Spatial-spectral analysis and adaptive binning method}

To optimize the binning of the X-ray data for spatially resolved study, we employ a state-of-the-art adaptive spatial binning method called the weighted Voronoi tessellations (WVT) binning algorithm (Diehl \& Statler 2006), which is a generalization of Cappellari \& Copin (2003) Voronoi binning algorithm. The

\footnotetext{
http://cxc.harvard.edu/ciao

2 https://heasarc.gsfc.nasa.gov/xanadu/xspec
} 

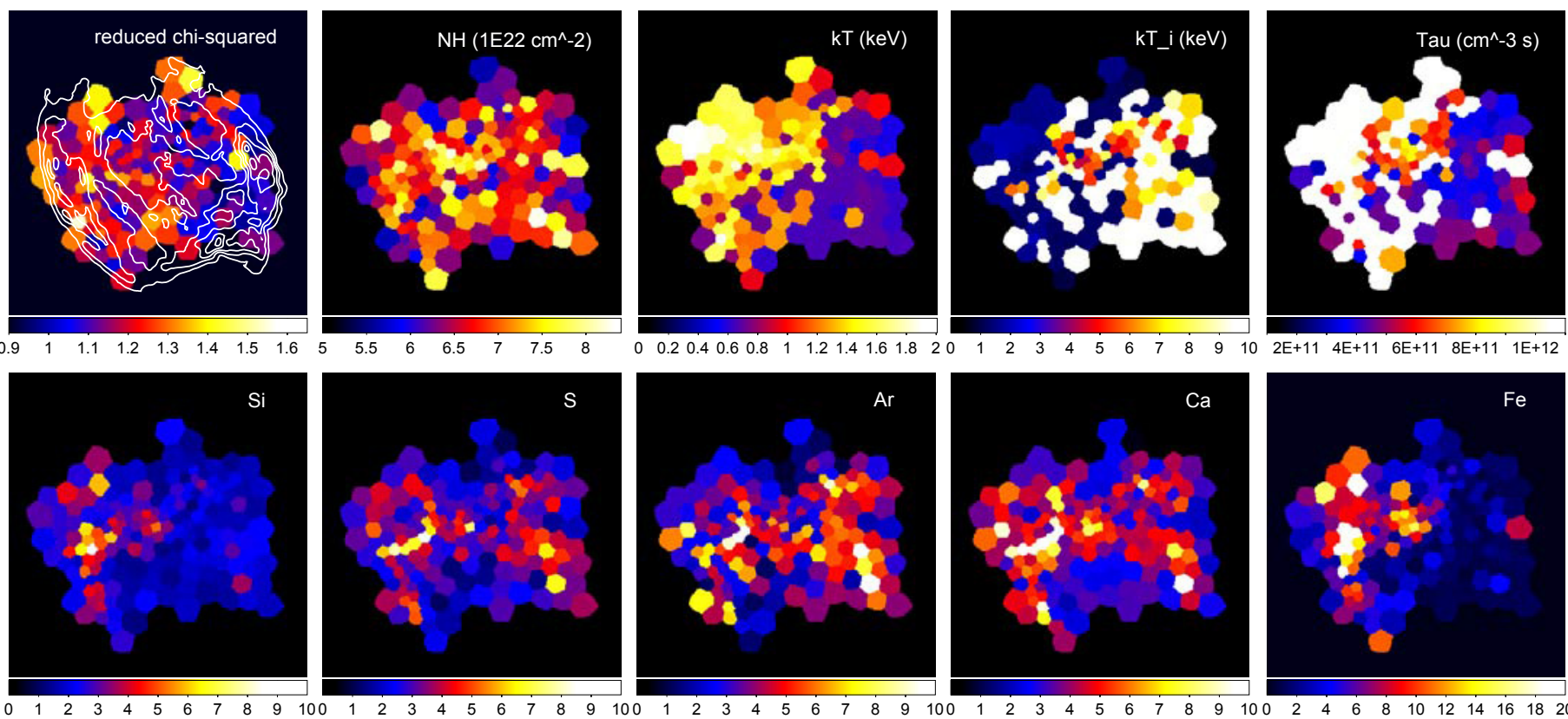

Fig. 1. Distribution of the parameters fitted with the best-fit single component model, which, for every cell, was taken to be either the absorbed "vrnei" or the absorbed "apec" model, whichever produced the smallest $\chi_{v}^{2}$. First panel: $\chi_{v}^{2}$ overlaid with VLA $20 \mathrm{~cm}$ radio contours (Helfand et al. 2006) in white color.

X-ray events taken from the event file are adaptively binned with the WVT binning algorithm.

First, we use the observation with longest exposure (obs. ID: 13440$)$ to generate the bins, with each bin containing about 3600 counts in $0.3-10 \mathrm{keV}$. Among the 238 detected bins, 177 are located inside the SNR, with mean counts per pixel $\left(1^{\prime \prime}\right)$ larger than 5. Subsequently, we extract spectra of the 177 regions from three Chandra data sets. The combined data provide $\sim 6000$ counts in each spatial bin. We then perform spectral analysis on the 177 bins associated with W49B by jointly fitting three groups of spectra from three observations. For each spatial bin and each observation, separate response matrix file and ancillary response file are generated. The background spectra are selected from a source-free region to the northeast of the SNR, which is at a similar Galactic latitude to the remnant.

Since previous studies (Kawasaki et al. 2005; Miceli et al. 2006, 2010; Ozawa et al. 2009; Lopez et al. 2013a,b) indicate that the spectrum of $\mathrm{W} 49 \mathrm{~B}$ is best fit with either a collisional ionization equilibrium (CIE) model or a recombining plasma model (a non-equilibrium ionization model with over-ionization), or a combination of the two models, we started our analysis by fitting each spatial bin twice, once with a CIE and once with an over-ionization model; in the end we selected the model that fits the spectra of a given spatial bin best with the smallest reduced chi-squared $\chi_{\nu}^{2}$; see Sect. 2.3).

Because this single-component model did not always give satisfactory fits, we decided to also use a two-component model, combining a relatively cool CIE component with a hotter component, which could be either a CIE or an over-ionized model (Sect. 2.4). The two component model gives better fits to the spectra, but a problem is that some of the parameters are correlated, so some additional constraints had to be imposed.

One should be aware that the recombining plasma model, with two more free parameters, may provide slightly smaller $\chi_{v}^{2}$ than the CIE model even though sometimes the two models are not statistically distinguished. Nevertheless, we found that for the single-component case discussed in Sect. 2.3 the two models can be distinguished in $90 \%$ of the extracted regions (based on the $F$-test, $2 \sigma$ level), and for the two-component case in Sect. 2.4, the two groups of models are distinguishable in $80 \%$ of regions.

\subsection{Single thermal component}

We jointly fit the spectra in each bin with an absorbed CIE model (vapec) and an absorbed recombining plasma model (vrnei) in Xspec, and select the model with smaller $\chi_{v}^{2}$ as the best-fit model. The two plasma models use the atomic data in the ATOMDB $\operatorname{code}^{3}$ version 3.0.7. The Tuebingen-Boulder interstellar medium (ISM) absorption model tbabs is used for calculation of the $\mathrm{X}$-ray absorption due to the gas-phase ISM, the grain-phase ISM, and the molecules in the ISM (Wilms et al. 2000). The vrnei model describes a plasma that has cooled/heated rapidly from an initial temperature $k T_{\mathrm{i}}$ to a temperature of $k T$, whereas the ionization state lags behind, and is characterized by a recombination/ionization timescale $\tau_{\mathrm{r}} / \tau_{\mathrm{i}}$ applied to all ions. When the ions are in the recombining (overionized) state, $k T_{\mathrm{i}}$ and the "ionization temperatures" of some ions $k T_{z}$ are larger than the current electron temperature $k T$. The lower limit on $k T_{\mathrm{i}}$ is set to $2 \mathrm{keV}$ to ensure that the recombining model is used, where the high ionization temperatures of $\mathrm{Ar}$ and $\mathrm{Ca}\left(k T_{z}=2.2-2.7 \mathrm{keV}\right.$; Kawasaki et al. 2005) are also considered. We set the upper limit of $k T_{i}$ to $10 \mathrm{keV}$, which is a very high value for the plasma in an evolved SNR. We allow the abundances of $\mathrm{Si}, \mathrm{S}, \mathrm{Ar}, \mathrm{Ca}$, and $\mathrm{Fe}$ to vary and tie the abundance of $\mathrm{Ni}$ to $\mathrm{Fe}$. Lower-mass elements, such as $\mathrm{O}, \mathrm{Ne}$, and $\mathrm{Mg}$, have X-ray line emission below $1.8 \mathrm{keV}$, in the part of the spectrum that is heavily affected by interstellar absorption. The abundances of these elements are therefore unconstrained by the fits, and we fixed the abundances to their solar values. The solar abundances of Asplund et al. (2009) are adopted in plasma emission and photoelectric absorption models. Compared to the older widely used abundances obtained by Anders \& Grevesse (1989), the $\mathrm{O}$ and Fe abundances

http://www. atomdb.org/ 

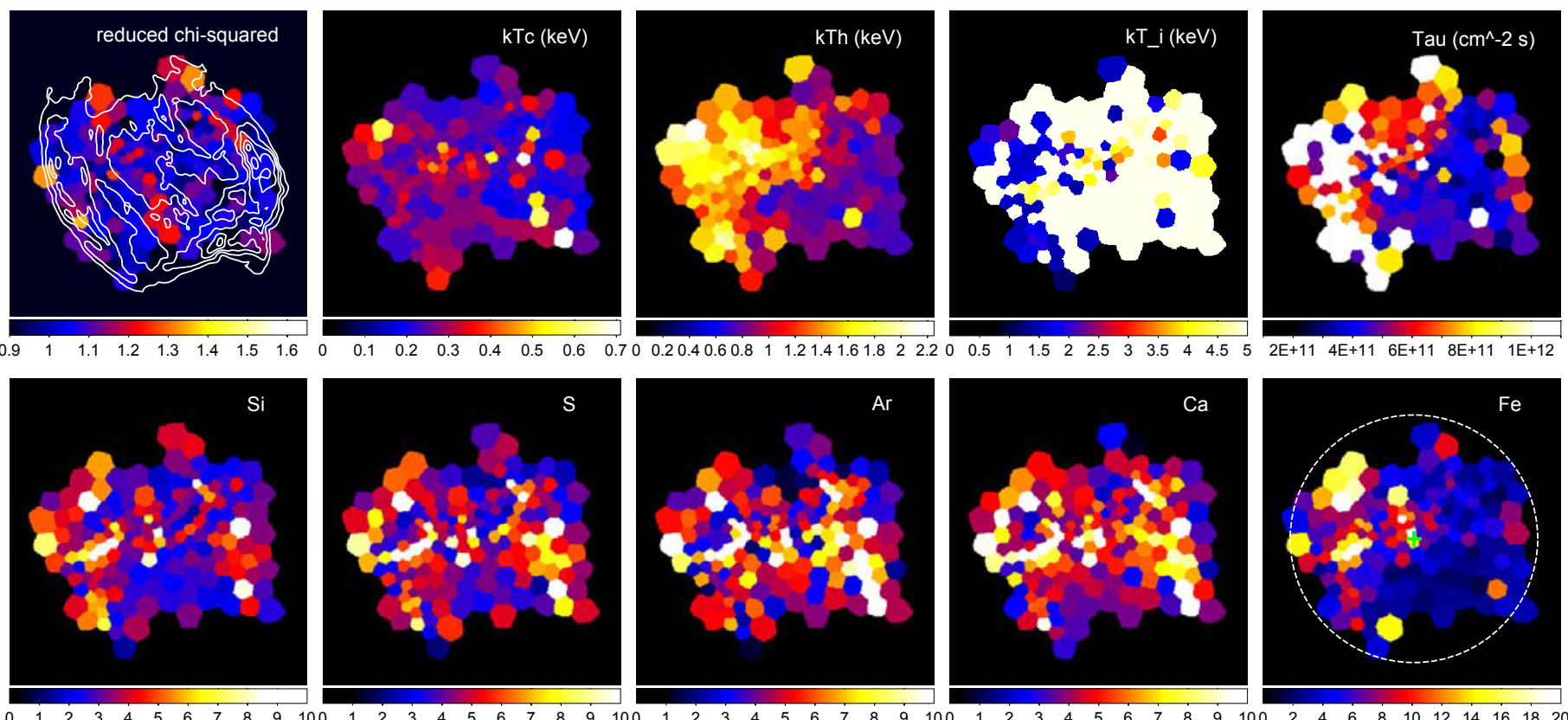

Fig. 2. Distribution of the parameters fitted with the best-fit double component model, which was either an absorbed "hot vrnei+cool apec" model, or an absorbed "hot vapec + cool apec" model. First panel: $\chi_{v}^{2}$ overlaid with VLA $20 \mathrm{~cm}$ radio contours in white color. Last panel: dashed circle and the green cross sign denote the SNR sphere used for density calculation and the sphere center used for abundance - PA diagram in Fig. 10, respectively.
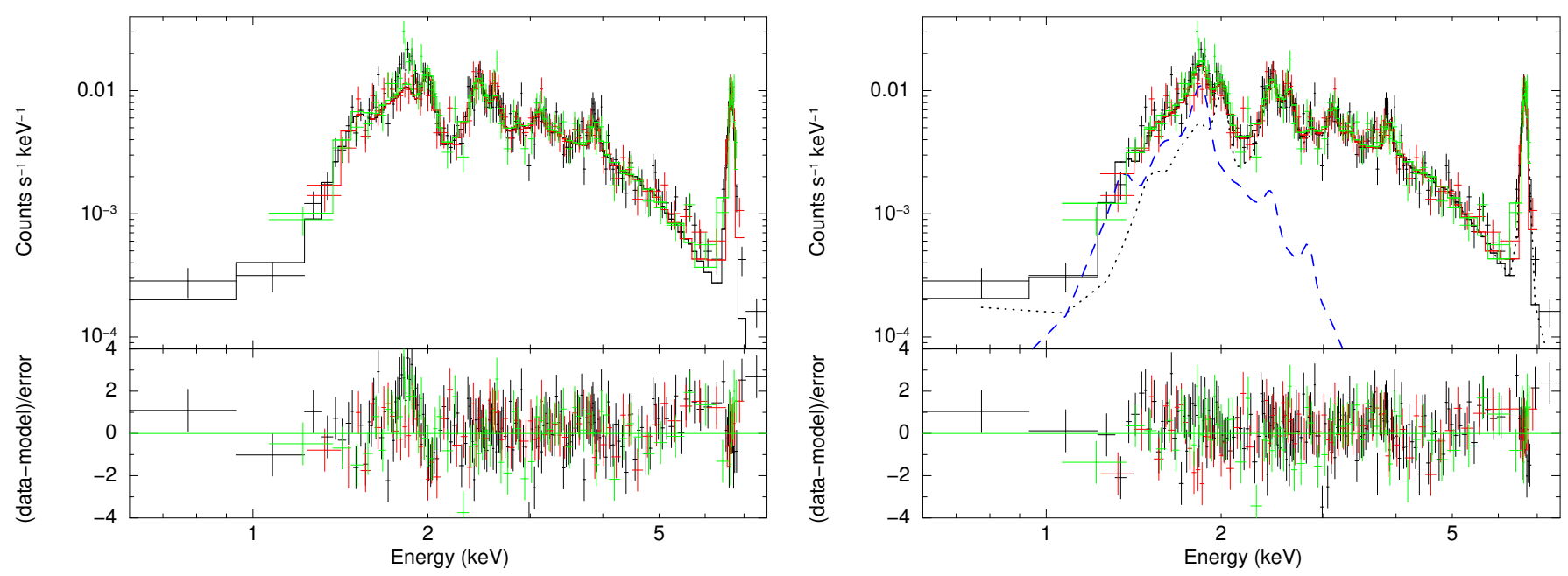

Fig. 3. Exemplified ACIS-S spectra from one bin in W49B fitted with one component (vapec; left: $\chi_{v}^{2} /$ d.o.f. $\left.=1.44 / 308\right)$ and two components $($ apec in blue dashed line plus vapec in black dotted line; right: $\chi_{v}^{2} /$ d.o.f. $\left.=1.15 / 307\right)$ models, respectively. The black, red, and green data correspond to the spectra from the observations 13440,13441 , and 117 , respectively.

in Asplund et al. (2009) decreased by $42 \%$ and $32 \%$, respectively. Therefore, adopting different solar abundances can result in differences to the obtained abundances (especially for Fe) and absorption column density ${ }^{4}$.

The single thermal component model gives a $\chi_{v}^{2}$ between 0.9 and 1.5 (degree of freedom (d.o.f.) $=222-365$; mean $\chi_{v}^{2} /$ d.o.f. $\left.=1.16 / 291\right)$ across the remnant. Figure 1 displays the spatial distribution of the best-fit parameters, including the foreground absorption $N_{\mathrm{H}}$, electron temperature $k T$, initial temperature $k T_{\mathrm{i}}$ (equal to $k T$ when CIE is the best-fit model), recombination timescale $\tau_{\mathrm{r}}$ (equal to the upper limit

4 For example, using the solar abundances of Anders \& Grevesse (1989) result in $\sim 30 \%$ smaller $N_{\mathrm{H}}$ and Fe abundance for the spectra showed in Fig. 3 (for both of the single and two component models). of $10^{14} \mathrm{~cm}^{-3} \mathrm{~s}$ when CIE is the best-fit model), and the abundances of $\mathrm{Si}, \mathrm{S}, \mathrm{Ar}, \mathrm{Ca}$, and $\mathrm{Fe}$. The figure illustrates a large variation of the gas properties inside $\mathrm{W} 49 \mathrm{~B}$. The $k T_{\mathrm{i}}$ values can be constrained in only a small fraction of regions, while in the southwestern regions they run to the upper limit of $10 \mathrm{keV}$.

\subsection{Two thermal components}

Previous studies suggest the existence of a cooler ISM component ( $\sim 0.25 \mathrm{keV}$; Kawasaki et al. 2005) in addition to the hot, metal-rich component (Hwang et al. 2000; Miceli et al. 2006; Lopez et al. 2013a). Moreover, the infrared observations reveal that a large amount of dense gas $\left(\sim 500 \mathrm{~cm}^{-3}\right.$ and $\left.\sim 800 M_{\odot}\right)$ is shocked by W49B (Zhu et al. 2014), which implies that the SNR in such an inhomogeneous environment should consist of 


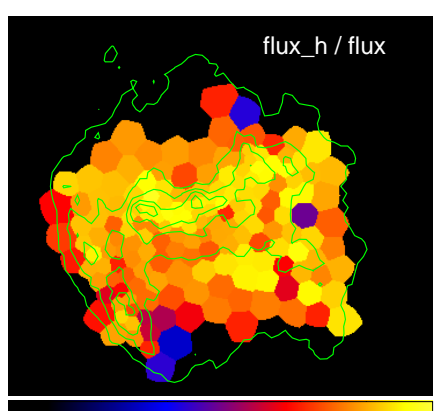

$\begin{array}{llllll}0.7 & 0.75 & 0.8 & 0.85 & 0.9 & 0.95\end{array}$

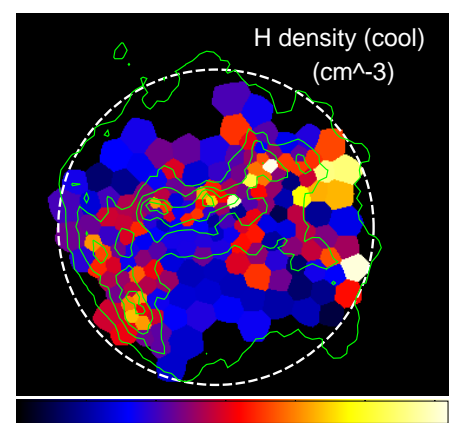

$\begin{array}{llllll}10 & 20 & 30 & 40 & 50 & 60\end{array}$

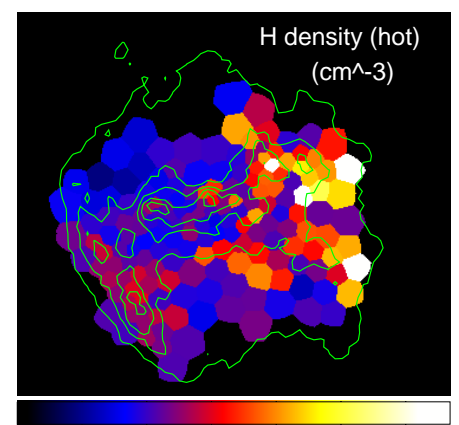

246

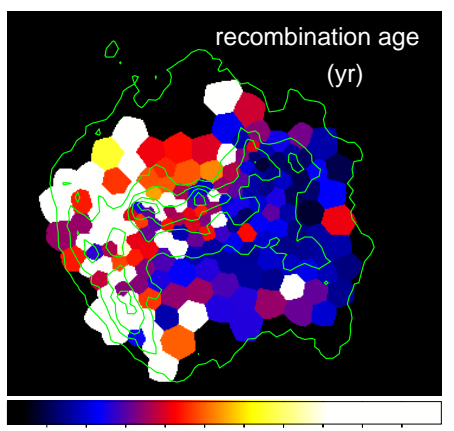

$2000 \quad 4000 \quad 6000 \quad 8000 \quad 10000$

Fig. 4. Distribution of the hot-to-total flux ratio (0.75-0.99), hydrogen densities in the cold and hot phases, respectively, and recombination age of the hot components, overlaid with green contours the Chandra X-ray emission in 0.3-10 keV. The dashed circle denotes the SNR sphere for the density calculation.

more than one density/temperature component. We, therefore also apply a two-thermal-component model to fit the spectra, in an attempt to check whether or not a cool component is needed across the SNR. The colder apec model with solar abundances is added to the absorbed vrneilvapec model and then used to fit the 177 groups of spectra. The soft X-ray photons $\lesssim 2 \mathrm{keV}$ (dominated by the cooler component) suffer heavy absorption, which results in some degeneracy between the cool component temperature $k T_{\mathrm{c}}$ and $N_{\mathrm{H}}$ in the spectral fit. We therefore assume that the foreground absorption of W49B is not changed across the small angular extent of the SNR $\left(<5^{\prime}\right)$ and fix the $N_{\mathrm{H}}$ value to $8 \times 10^{22} \mathrm{~cm}^{-2}$. This value is close to the bestfit $N_{\mathrm{H}}$ for the global spectra, and is also similar to the mean $N_{\mathrm{H}}$ value if $N_{\mathrm{H}}$ is allowed to vary for the 177 spectra. The upper limit for $k T_{\mathrm{c}}$ is set to $0.7 \mathrm{keV}$ to ensure that it is smaller than $k T_{\mathrm{h}}$.

Figure 2 shows the detailed distribution of the gas properties in W49B based on the two-component model, including $\chi_{v}^{2}$, the temperatures of the colder and hotter component $\left(k T_{\mathrm{c}}\right.$ and $k T_{\mathrm{h}}$, respectively), $k T_{\mathrm{i}}, \tau_{\mathrm{r}}$, and the abundances of $\mathrm{Si}, \mathrm{S}, \mathrm{Ar}, \mathrm{Ca}, \mathrm{Fe}$ elements. The Si distribution is smoother in the two-thermalcomponent model. Here the upper limit of $k T_{\mathrm{i}}$ is set to $5 \mathrm{keV}$, which appears to be a limit to the electron temperatures found in young SNRs (Vink 2012). We note that the proton temperature may be higher, but the ionization properties of the plasma are determined by the electron temperatures. Moreover, the adopted upper limit value is indicated by the best-fit $k T_{\mathrm{i}}$ values $(\sim 5 \mathrm{keV})$ in the SNR center.

Although the overall temperature and abundance patterns obtained with single and double component models appear to be similar, the two-thermal-component model better describes the spectra than the model with only one thermal component: it results in smaller $\chi_{v}^{2}$ values (mean value of 1.06 and d.o.f. of 290), as well as smaller residuals in the spectral fit for the Si-XIII line emission for the range $1.8-2.2 \mathrm{keV}$ (Fig. 3). The cooler component mainly affects the soft spectra $\lesssim 2 \mathrm{keV}$ and the best-fit values of Si (see Fig. 3 for an example of the spectra fitted with one component and two components models, respectively). It is statistically meaningful to add the extra thermal component to the single-thermal-component model to improve the spectral fit of majority regions according to an $F$-test analysis (mean null hypothesis probability $10^{-7}$, adopting a mean d.o.f. of 291 and $\chi_{v}^{2}=1.16$ in the single thermal model). For $92 \%$ of the bins, the two-component model significantly improves the fit with an $F$-test probability less than the typical value of 0.05 ( $2 \sigma$ level), while for the other $8 \%$ bins near the western boundary the improvement is less significant.
The X-ray flux of W49B is dominated by the hot component, as indicated by the large fraction of the hot-component flux to the total flux in $0.5-8 \mathrm{keV}$ (flux_h/flux $=0.78-0.99$; see the left panel of Fig. 4). The X-ray contribution from the cool component (1-flux_h/flux) is larger near the SNR shell than in the SNR interior. As a result, the single component fit with $N_{\mathrm{H}}$ free gives smaller $N_{\mathrm{H}}$ values near the SNR edge to compensate the flux of the missing cool component (see the $N_{\mathrm{H}}$ distribution in Fig. 1). Therefore, the variation of $N_{\mathrm{H}}$ is likely to be much smaller than suggested by the single-component model.

A temperature gradient for the hot component gas $\left(k T_{\mathrm{h}}\right)$ is revealed with an orientation northeast to southwest (see Fig. 2). The $k T_{\mathrm{h}}$ is as high as $\sim 2.2 \mathrm{keV}$ in the northeast but decreased to $\sim 0.7 \mathrm{keV}$ in the southwest. The $k T_{\mathrm{c}}$ shows some variation across the SNR with a mean value of $0.27 \mathrm{keV}$. In a small fraction of regions with $N_{\mathrm{H}}$ deviated largely from $8 \times 10^{22} \mathrm{~cm}^{-2}, k T_{\mathrm{c}}$ can be affected by $N_{\mathrm{H}}$ due to the degeneracy between them. For example, a region in the southwest shows large $k T_{\mathrm{c}}=0.7 \mathrm{keV}$, which could be reduced to $0.18 \mathrm{keV}$ if $N_{\mathrm{H}}$ is free $\left(\sim 10^{23} \mathrm{~cm}^{-2}\right)$.

The recombining plasma appears to be present throughout the SNR, except in the southeast shell, and occupies about three quarters of the studied area. The recombination timescale varies in the range $1-10 \times 10^{11} \mathrm{~cm}^{-3} \mathrm{~s}$. The recombining gas and CIE gas can be distinguished by comparing its $k T_{\mathrm{i}}$ and $k T_{\mathrm{h}}$ or by checking $\tau_{\mathrm{r}}$ values: the recombining gas shows $k T_{\mathrm{i}}>k T_{\mathrm{h}}$ and $\tau_{\mathrm{r}} \lesssim 10^{12} \mathrm{~cm}^{-3} \mathrm{~s}$. The CIE region is colored in white in the $\tau_{\text {r }}$ panel of Fig. 2 (upper-right). Our study confirms the existence of recombining plasma in the center and west as pointed out by previous studies (e.g., Miceli et al. 2010; Lopez et al. 2013a). We note that the recombining plasma is also patchily distributed in the northern hemisphere of the remnant, but here the recombination timescales are generally much longer than in the southwest.

\subsection{Density and mass of the shocked gas}

W49B has a centrally bright X-ray morphology, indicating an enhanced plasma density in the interior, unlike the shell-type SNRs in Sedov phase with a density enhancement at the shells (Borkowski et al. 2001). Since the SNR is highly structured without a clear understanding of its three-dimensional density distribution, any complicate hypothesis on the gas distribution (e.g., bar + shell, disk + shell) could differ from the real distribution and introduce unknown uncertainties. Therefore, we take the simplest assumption that the plasma is uniform in each bin, so as to provide a mean gas density of the X-ray-emitting gas. We 
note that the following results are based on this oversimplified assumption of the geometry.

We estimate the mean density $n_{\mathrm{H}}$ for a given bin using the normalization parameter in Xspec (norm $=10^{-14}$ / $\left(4 \pi d^{2}\right) \int n_{e} n_{\mathrm{H}} d V$, where $d$ is the distance, $n_{e}$ and $n_{\mathrm{H}}$ are the electron and $\mathrm{H}$ densities in the volume $V ; n_{e}=1.2 n_{\mathrm{H}}$ for fully ionized plasma with solar or enhanced metal abundances in W49B) and an assumed prism geometry for each bin. Each prism has an area of the region and a depth across the SNR $l(r)=2 \sqrt{R_{\mathrm{S}}^{2}-r^{2}}$, where the radius of the SNR is $R_{\mathrm{S}}=2 ! 2$, corresponding to $6.4 \mathrm{pc}$ at a distance of $10 \mathrm{kpc}$, and $r$ is its projection distance to the assumed SNR center. The SNR circle denoted in Fig. 4 encloses all regions, except a bin in the southwest, where its density and mass are not calculated. The two-temperature gas is assumed to fill the whole volume $\left(f_{\mathrm{c}}+f_{\mathrm{h}}=1\right)$ and in pressure balance $\left(n_{\mathrm{c}} T_{\mathrm{c}}=n_{\mathrm{h}} T_{\mathrm{h}}\right)$, where $f$ and $n$ are the filling factor and hydrogen density, respectively, and the subscripts "c" and "h" denote the parameters for the cool and hot phases, respectively.

The distributions of the densities are displayed in Fig. 4. The brightness of the X-ray emission in $0.3-10 \mathrm{keV}$ is overlaid with contours for comparison purposes. The hydrogen density $n_{\mathrm{c}}$ in the cooler component gas is clearly enhanced along the "barlike" feature across the SNR center and the shells in the eastern and western sides; it displays a good spatial correlation with the X-ray brightness. An enhancement of the density in those regions is also present in the hot component. The mean hydrogen density of the colder and hotter components are $24 \mathrm{~cm}^{-3}$ and $5 \mathrm{~cm}^{-3}$, respectively, and the mean filling factor of the hotter phase is $40 \%$. The total masses of the X-ray-emitting gas are $M_{\mathrm{c}}=484_{-34}^{+41} d_{10}^{2.5} M_{\odot}$ for the cool phase and $M_{\mathrm{h}}=52 \pm 8 d_{10}^{2.5} M_{\odot}$ for the hot phase, where $d_{10}=d /(10 \mathrm{kpc})$ is the distance scaled to $10 \mathrm{kpc}$.

The mass uncertainties are calculated by using the $90 \%$ uncertainties of the normalization parameters and the filling factors of the 176 bins, where the uncertainties in the filling factors are incorporated into the overall error estimates, also taking into account uncertainties in the temperatures and normalizations of the two components. If the errors are asymmetric, we take the largest error. The systematic uncertainty is dominated by assumptions about the volume $V$ or the depth $l$ of the X-ray emitting gas, since the gas mass in bin $i$ weakly depends on them $\left(M(i) \propto V(i)^{1 / 2} \propto l(i)^{1 / 2}\right.$ with given distance and norm $)$. Since mixed-morphology SNRs generally have a relatively smooth radial density distribution, we assume the X-ray-emitting gas fills the whole SNR from front to back. This introduces some uncertainty, as the real depth, $l(i)$, can be smaller. In the extreme case that the X-ray emission is only arising from a thin shell with a thickness of $1 / 12 R_{\mathrm{S}},{ }^{5}$ the masses are $202_{-14}^{+18}$ and $21 \pm 3 M_{\odot}$ in the cool and hot components, respectively, which puts very conservative lower limits on the gas masses in W49B. We note that the thin shell geometry does not agree with the overall, center-filled, X-ray morphology of W49B.

$M_{\mathrm{c}}$ is much larger than what could have been produced by the progenitor wind or the ejecta. Hence, the cool component is dominated by the heated interstellar gas. The mass of the hot component is also too large to correspond to the total supernova ejecta mass, which suggests that it consists of a mixture of ejecta and circumstellar material. An alternative could be

\footnotetext{
5 For a uniform density and a shock compression ratio of four, mass conservation suggests that for shell-type SNR the shell should have a thickness of approximately one twelfth $R: 4 \pi R^{2} \Delta R\left(4 \rho_{0}\right)=4 \pi / 3 R^{3} \rho_{0}$.
}

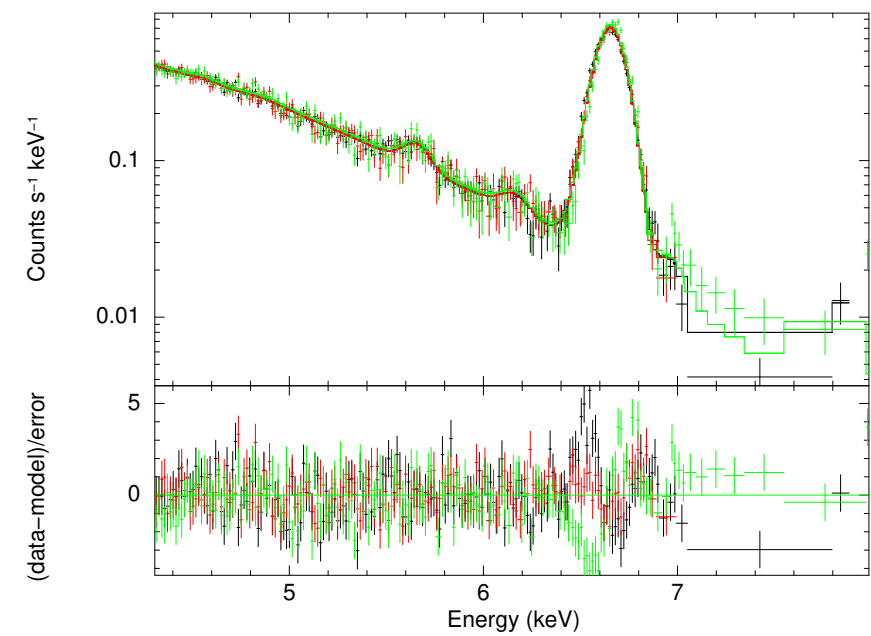

Fig. 5. Global spectra of $\mathrm{W} 49 \mathrm{~B}$ in the $4.3-8.0 \mathrm{keV}$ range fitted with a recombining plasma model vvrnei. The black, red, and green lines correspond to the spectra from observations 13440,13441 , and 117, respectively.

that the hot component consists of almost pure ejecta material, with almost no hydrogen. In that case, the bremsstrahlung continuum is also dominated by electron-metal interactions rather than electron-proton interactions. The high average charge of the atoms and the enhanced ratio of electrons to atom results then in a higher emissivity per atom, which results in a lower mass for the hot component (Vink 2012, Sects. 6.1 and 10.3). Abundance ratios are not very sensitive to whether the gas is metal-pure, or hydrogen-rich with enhanced metal abundances. Currently with CCD X-ray spectroscopy we cannot distinguish between a pure metal plasma and a hydrogen-rich plasma with enhanced abundances. However, in the future, high-resolution imaging spectroscopy with the XARM (X-ray Astronomy Recovery Mission) and/or Athena could perhaps distinguish these two cases spectroscopically. Given the age of W49B (see also below) a mixture of hydrogen-rich and metal-rich ejecta seems the most likely scenario.

\subsection{Abundance and distribution of the ejecta}

The abundances and distribution of heavy elements have played an important role in probing the explosion mechanism of SNRs. We obtained average abundances $[\mathrm{Si}]=3.4_{-0.7}^{+0.8},[\mathrm{~S}]=4.9_{-1.0}^{+1.1}$, $[\mathrm{Ar}]=5.1_{-1.0}^{+1.2},[\mathrm{Ca}]=5.2_{-1.0}^{+1.2}$, and $[\mathrm{Fe}]=5.7_{-1.1}^{+1.2}$. The average abundance values here are based on the weighted sum (by the estimated gas mass for each sub-region) over all individual subregions, and are insensitive to the emission volume assumption as long as the emission volume does not vary sharply at different regions. The abundance of $\mathrm{Fe}$ is greatly enhanced in the east of the SNR, while the $\mathrm{Si}, \mathrm{S}, \mathrm{Ar}, \mathrm{Ca}$ elements are highly enhanced across the SNR, especially along the east and west (nearly axial symmetric distribution; see Sect. 3.2.2).

W49B is the first cosmic source in which $\mathrm{Cr}$ and possibly $\mathrm{Mn}$ emission were found (Hwang et al. 2000). The global spectra of W49B in $4.3-8.0 \mathrm{keV}$ show a clear $\mathrm{Cr}$ line at $\sim 5.6 \mathrm{keV}$ and a Mn bump at $\sim 6.1 \mathrm{keV}$ (see Fig. 5). We fit the global spectra with the vvrnei model and obtained $[\mathrm{Cr}]=6.6 \pm 0.8,[\mathrm{Mn}]=12.5 \pm 2.7$ (see Fig. $5 ; \chi_{v}^{2} /$ d.o.f. $=1.74 / 527 ; k T=1.48_{-0.01}^{+0.07} \mathrm{keV}, N_{\mathrm{H}}$ is fixed to $8 \times 10^{22} \mathrm{~cm}^{-2}, k T_{\mathrm{i}} \geq 4.6 \mathrm{keV},[\mathrm{Fe}]=3.2 \pm 0.1$; [Ni] cannot be constrained and thus tied to $[\mathrm{Fe}] ; \tau_{\mathrm{r}}=6.0_{-0.6}^{+1.1} \times 10^{11} \mathrm{~cm}^{-3} \mathrm{~s}$ ). The thermal plasma model constrains the Mn $\mathrm{K}$ line flux to $1.6 \times 10^{-5} \mathrm{~cm}^{-2} \mathrm{~s}^{-1}$, similar to that obtained by Miceli et al. 


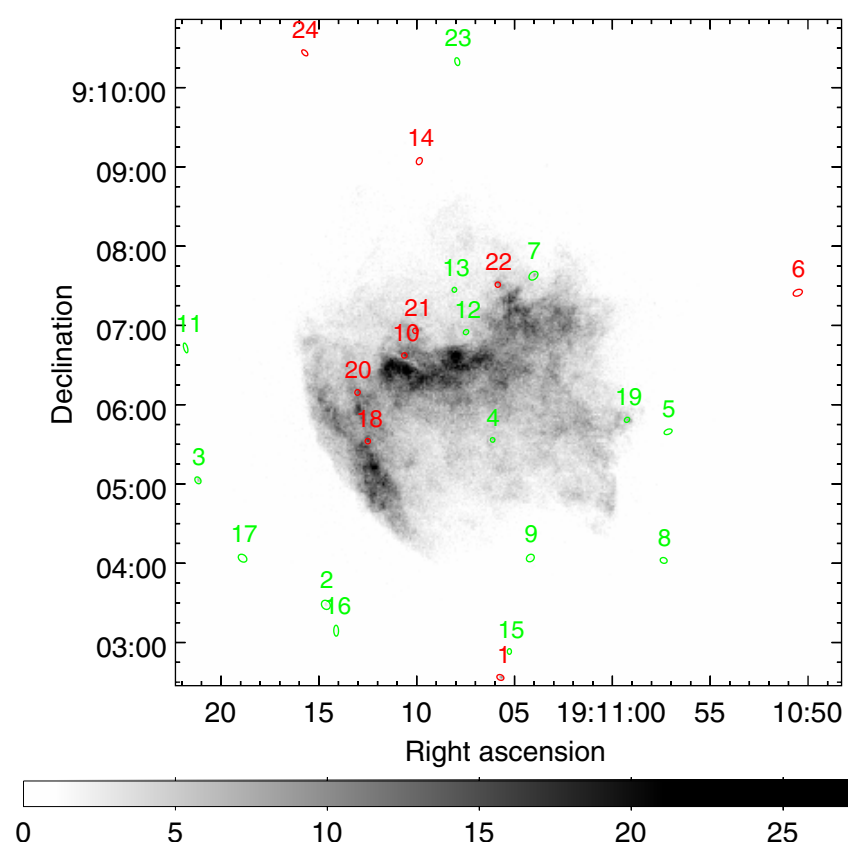

Fig. 6. Detected point-like sources in the Chandra $0.7-5 \mathrm{keV}$ raw image of W49B. The color-bar shows the scale of the counts per pixel (0' 5$)$. The details of the sources in the ellipses are summarized in Table A.1. The red regions denote the sources with best-fit blackbody luminosity in the range $7.0 \times 10^{32}-2.7 \times 10^{34} d_{10}^{2} \mathrm{erg} \mathrm{s}^{-1}$ corresponding to the luminosity range of NSs at the age of 5-6 kyr predicted with the minimal cooling paradigm (see Appendix A and Fig. 8 for details).

(2006) and Yang et al. (2013). The CIE model gives a slightly larger $\chi_{v}^{2}(1.78)$, with $[\mathrm{Cr}]=5.9 \pm 0.7,[\mathrm{Mn}]=13.9_{-4.1}^{+4.3}$. Hereafter, we use the best-fit abundance results of the vvrnei model. The residuals at around $6.6 \mathrm{keV}$ are likely due to the gain shifts for ACIS, but the shifts are within typical $0.3 \%$ systematic uncertainties in the ACIS gain (response from the CXC calibration scientists $)^{6}$. The Fe abundance obtained by fitting the global spectra is smaller than the mass-weighted abundance $\left(5.7_{-1.1}^{+1.2}\right)$, since the Fe element and the plasma properties vary across the SNR (see Fig. 2).

\subsection{Point-like sources in the vicinity}

W49B has been considered to host a black hole, as no potential NS was detected down to an X-ray luminosity of $2.7 \times 10^{31} \mathrm{erg} \mathrm{s}^{-1}$, and a core collapse origin was assumed (Lopez et al. 2013b). However, the remnant reveals a very nonuniform X-ray brightness, which provides a spatially varied background for point-source detection. After considering the bright background from the SNR plasma, we detected 24 point-like sources in, or in the vicinity of, W49B using the Mexican-Hat wavelet detection method (wavedetect in CIAO; see Fig. 6). The point spread function (PSF) of the chip and the vignetting effect are taken into account. The applied significance threshold of source identification $\left(10^{-6}\right)$ corresponds to one false detection in the image.

We derived the blackbody temperatures and luminosities of the sources based the photon fluxes $f$ and hardness ratios (see Fig. 7). The luminosities of these sources are in the range $10^{32.1-36.2} \mathrm{erg} \mathrm{s}^{-1}$ at an assumed distance of $10 \mathrm{kpc}$. The details of

6 http://cxc.harvard.edu/cal/summary/Calibration Status_Report.html

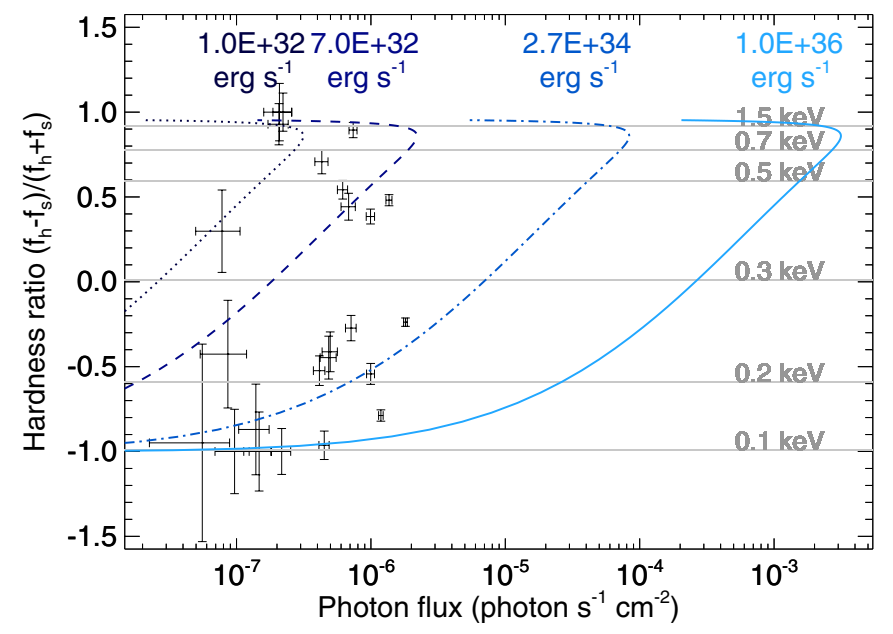

Fig. 7. Hardness ratios (see the definition in Appendix A) and photon fluxes of the 24 point-like sources as a function of the blackbody temperature and luminosity at a distance of $10 \mathrm{kpc}$. The curved lines from the left to right indicate the luminosity from $10^{32}$ to $10^{36} \mathrm{erg} \mathrm{s}^{-1}$. An absorption of $N_{\mathrm{H}}=8 \times 10^{22} \mathrm{~cm}^{-2}$ is assumed for all the sources.

the detection method and analysis are elaborated in Appendix A. Detailed information about these sources is summarized in Table A.1.

\section{Discussion}

Here we revisit the progenitor problems and discuss the $\mathrm{CC}$ (normal and energetic) and Type-Ia scenarios mainly based on three properties: metal abundances, metal distributions, and environment. The metal abundances can be compared to predictions of supernova nucleosynthesis models, the distribution of metals reveals explosion (a)symmetries, and the density distribution provides information on the circumstellar environment. We will also examine the SNR properties and discuss the origin of the bar-like morphology according to the spatially resolved spectroscopic analysis.

\subsection{CC scenario and its problems}

\subsubsection{Cavity}

W49B is suggested to be interacting with a molecular cavity surrounding the remnant (Chen et al. 2014; Zhu et al. 2014). A massive star can evacuate a hot, low-density bubble with its strong stellar wind during the main sequence stage. The slow and dense wind during its later red supergiant stage will, however, in most cases not reach the main sequence cavity shell. In the molecular environment with a typical pressure of $p / k \sim 10^{5} \mathrm{~cm}^{-3} \mathrm{~K}$ (Chevalier 1999; Blitz 1993), the maximum radius of the bubble $R_{b}$ is determined by the progenitor mass $M_{*}: R_{b} \approx 1.22 M_{*} / M_{\odot}-9.16 \mathrm{pc}$ (Chen et al. 2013). This linear relation is valid for stars with masses $<25 M_{\odot}$, while beyond the mass the bubble could be larger due to the contribution from the fast wind in Wolf-Rayet stage. If the molecular cavity surrounding $\mathrm{W} 49 \mathrm{~B}$ was created by the main sequence wind of a massive progenitor, a bubble with a radius of 6.4 would suggest a progenitor mass of $\sim 13 M_{\odot}$ (see also Chen et al. 2014; Zhang et al. 2015). A smaller bubble size ( $\sim 5 \mathrm{pc})$ is also likely as suggested by Keohane et al. (2007), and indicates a progenitor mass $\lesssim 13 M_{\odot}$. This is inconsistent with the idea that the supernova explosion resulted in the creation of a black hole 


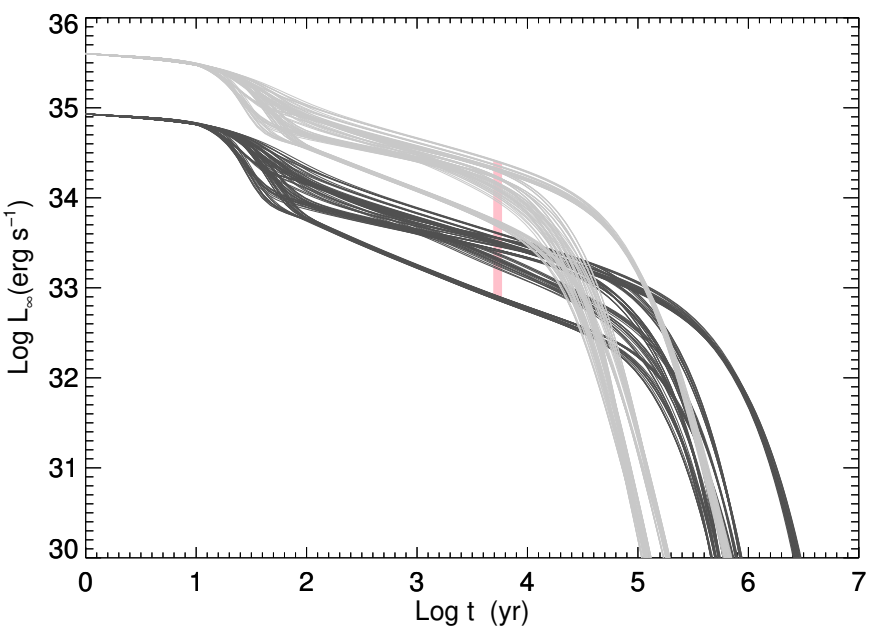

Fig. 8. Predictions of NS cooling according to the minimal cooling paradigm (see Page et al. 2009). The light- and dark-gray lines represent the cooling curves of NSs with light- and heavy-element envelopes, respectively. The curves for intermediate-element envelopes are between them. Each envelope group includes 100 curves corresponding to four choices of ${ }^{3} P_{2}$ gaps, and five choices of neutron ${ }^{1} S_{0}$ and proton ${ }^{1} S_{0}$ gaps. The pink belt indicates the luminosity range of an NS in an age range of 5-6 kyr.

(Lopez et al. 2013a), which is considered to require a $>25 M_{\odot}$ progenitor. A $>25 M_{\odot}$ progenitor instead would create a wind bubble in the molecular cloud of $\sim 21 \mathrm{pc}$.

\subsubsection{Missing compact object?}

A massive single star with $M_{*} \lesssim 20-25 M_{\odot}$ ends its life with a NS (Heger et al. 2003). The NSs, which are born extremely hot $\left(>10^{10} \mathrm{~K}\right)$, cool predominantly via neutrino emission from the interior for $\sim 10^{4}-10^{5} \mathrm{yr}$ after birth. The cooling curve or luminosity-age relation depends sensitively on the equation of state of dense matter, NS mass, and envelope composition. Page et al. (2004, 2009) proposed the minimal cooling scenario including Cooper pair breaking and formation process to explain the observed luminosities of considerably young NSs. According to the minimal cooling scenario, young NSs radiate X-ray emission in the initial few kyr and their temperatures and luminosities are a function of the NS age.

Figure 8 shows the cooling curve (luminosity evolution) of NSs based on the minimal cooling paradigm, ${ }^{7}$ which assumes that no enhanced neutrino emission is allowed in NSs (see Page et al. 2009). All models are for $1.4 M_{\odot}$ stars built using the NS equation of state of Akmal et al. (1998). The model predicts a luminosity range of $L_{\infty}=7.0 \times 10^{32}-2.7 \times 10^{34} \mathrm{erg} \mathrm{s}^{-1}$ for the NS at an age of W49B (5-6 kyr; see discussion in Sect. 3.4).

Nine of the 24 detected point-like sources are in the luminosity range predicted for an NS at an age of 5-6 kyr according to the minimal cooling paradigm (regions labeled in red in Fig. 6; distance of $10 \mathrm{kpc}$ is assumed; between the dashed and dot-dashed curves in Fig. 7). We note that some of the sources may not be real point sources, but compact clumps of gas associated with W49B, while sources outside the SNR boundary would need a large transverse velocity $\left(\gtrsim 10^{3} \mathrm{~km} \mathrm{~s}^{-1}\right)$ to establish a connection with the SNR. As all the point sources are off-center, the NS

\footnotetext{
7 Cooling code from Dany P. Page: http://www . astroscu. unam. $\mathrm{mx} /$ neutrones/NSCool/
}

may have been born with a high velocity $\left(>400 \mathrm{~km} \mathrm{~s}^{-1}\right.$ assuming an SNR age of $6 \mathrm{kyr}$ ) if W49B was caused in a CC explosion. Therefore, even if W49B is a CC SNR, there is still the possibility that it contains a cooling NS, rather than a black hole, as suggested by Lopez et al. (2013b).

\subsubsection{Metal abundances and yields}

For a CC SNR showing super-solar abundances, the progenitor mass could be estimated by comparing the abundance ratios with those predicted by the nucleosynthesis models, since the nucleosynthesis yields of CC SNe are related to the progenitor mass, stellar metallicity (solar assumed in this study), and explosion energy (e.g., Nomoto et al. 2006). Figure 9 shows the comparison of the abundances of $\mathrm{Si}, \mathrm{Ar}, \mathrm{Ca}, \mathrm{Cr}, \mathrm{Mn}$, and $\mathrm{Fe}$ relative to Ar with the predictions of the spherical supernova explosion model (Sukhbold et al. 2016), hypernova model (Nomoto et al. 2006), and bipolar explosion model (Maeda \& Nomoto 2003). The observed abundance ratios are ascending with the atomic weight from $\mathrm{Si}$ to $\mathrm{Mn}$. We found that none of the available nucleosynthesis models explain the ascending abundance ratios. The less massive stars $\left(12-13 M_{\odot}\right)$ provide a flatter abundance pattern as function of element mass, whereas the very massive progenitors and energetic models produce sharper descending abundance ratio patterns (less IGEs), which are more deviated from the observed ratios.

Besides the inconsistent abundance ratios, none of the abovementioned CC models explain the observed amount of IGEs. The $\mathrm{Cr}, \mathrm{Mn}$, and $\mathrm{Fe}$ ejecta masses in the hotter-phase gas are $M_{\mathrm{Cr}}=4.9 \pm 1.1 \times 10^{-3} M_{\odot}, M_{\mathrm{Mn}}=6.6 \pm 1.9 \times 10^{-3} M_{\odot}, M_{\mathrm{Fe}}=$ $0.32_{-0.09}^{+0.10} M_{\odot}$, respectively. The nucleosynthesis models used in this study predict higher iron-group production for the stars with higher masses. A $25.2 M_{\odot}$ star produces $6.4 \times 10^{-4} M_{\odot}$ of $\mathrm{Cr}, 3.2 \times 10^{-4} M_{\odot}$ of $\mathrm{Mn}$, and $0.048 M_{\odot}$ of $\mathrm{Fe}$ in a spherical explosion, each of which is about an order of magnitude lower than the value obtained from X-ray observations. A $25 M_{\odot}$ undergoing energetic $\left(10^{52} \mathrm{erg}\right)$ spherical explosion produces slightly more $\mathrm{Cr}$, $\mathrm{Mn}$, and $\mathrm{Fe}\left(0.001 M_{\odot}, 3.5 \times 10^{-4} M_{\odot}\right.$, and $0.007 M_{\odot}$, respectively) compared to normal explosion energies, but this remains insufficient to create the observed yields. Similarly, all of the bipolar CC explosion models predict much smaller IGE yields than the observed values. The bipolar energetic explosion of a $25 M_{\odot}$ star (model $25 \mathrm{~A}$ ) produces $\mathrm{Cr}, \mathrm{Mn}$ and Fe masses of $7.5 \times 10^{-4} M_{\odot}, 2.3 \times 10^{-4} M_{\odot}$, and $0.082 M_{\odot}$, respectively.

Hence, the CC models fail to explain the observed abundance ratios and under-predict the mass of IGEs. Due to the large abundances of $\mathrm{Cr}$ and $\mathrm{Mn}$, this conclusion is unchanged, even taking into account the large systematic uncertainties in $M_{\mathrm{h}}\left(>18 M_{\odot}\right.$; see Sect. 2.4).

\subsection{Aspherical Type-la explosion}

\subsubsection{Metal abundances and yields}

Type-Ia SNe are the dominant factories of IGEs (see a recent review of Seitenzahl \& Townsley 2017). The large masses of $\mathrm{Cr}$, $\mathrm{Mn}$, and $\mathrm{Fe}$ and the high IGE/intermediate-mass element (IME) ratio in W49B clearly suggest a Type-Ia origin. As shown in the bottom panels of Fig. 9, we compare the abundances ratios of the SNR to the predicted results of different Type-Ia SNe models, including DDT models followed with spherical or extremely off-center slow flagrations, according to the three-dimensional model from Seitenzahl et al. (2013b) and two-dimensional models from Maeda et al. (2010b). The classical deflagration model 

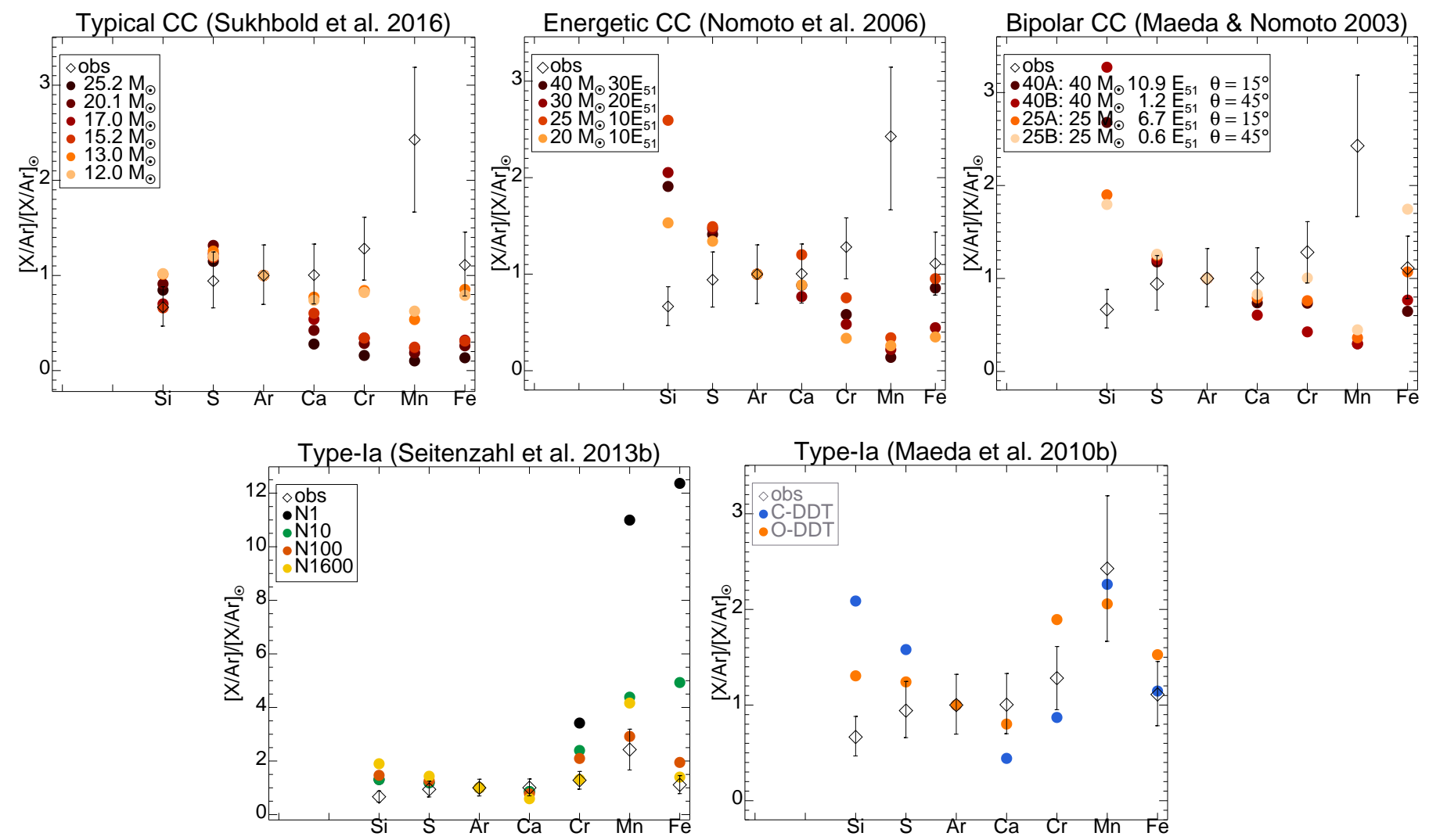

Fig. 9. Abundances of $\mathrm{Si}, \mathrm{Ar}, \mathrm{Ca}, \mathrm{Cr}, \mathrm{Mn}$, and Fe relative $\mathrm{Ar}$ (with error bars) compared with the predictions of supernova nucleosynthesis models. Upper panels: CC spherical explosion models (left), and energetic spherical (middle) and bipolar explosion models (right; the explosion energy are $10-30 \times 10^{51} \mathrm{erg}, E_{51}$ stands for $10^{51} \mathrm{erg}, \theta$ is the opening half-angle of the jet) for different progenitor masses. Bottom-left panel: three-dimensional DDT Type-Ia models, where the deflagration is ignited in 1, 10, 100, and 1600 spherical sparks, respectively, near the WD center. Bottom-right panel: compares the DDT models which followed the two-dimensional spherical deflagration (C-DDT) and extremely off-center deflagration (O-DDT) ignitions, respectively. Solar progenitor metallicity is used for all models.

W7 (Nomoto et al. 1984) was excluded since it over-predicts the Fe abundance.

For the models in the bottom-left panel of Fig. 9, the deflagration is assumed to be ignited from different numbers of sparks in a WD with a central density of $2.9 \times 10^{9} \mathrm{~g} \mathrm{~cm}^{-3}$. The model $\mathrm{N} 1$ is for the single spot ignition and N100 denotes the 100-spot ignition. The occurrence of multi-spot ignitions, which covers a range of offset radii, is a probable consequence of the turbulent convection of the WD prior to the thermonuclear runaway (Garcia-Senz \& Woosley 1995; Woosley et al. 2004; Iapichino et al. 2006). The fewer sparks burn less materials to power the expansion of the WD and thus the deflagration is weaker. The moderately strong expansion causes a high central density at the onset of detonation, and most of the remaining fuel is burned to IGEs by detonation. Conversely, a larger number of ignition kernels produces relatively more IMEs during incomplete burning (Seitenzahl et al. 2013b).

The abundance ratios in $\mathrm{W} 49 \mathrm{~B}$ can be well described by the multi-spark ignition models N100 and N1600, except for the element $\mathrm{Si}$. Hence, the model with moderate to large numbers of ignition sparks reproduces the abundances and yields in W49B well, whereas the fewer ignition sparks result in Fe/IME ratios that are too high.

\subsubsection{Aspherical explosion}

The ejecta distribution in W49B is not spherical, as shown in the abundance-position angle (PA) diagram (Fig. 10). Here the explosion center is assumed at the approximate geometric center

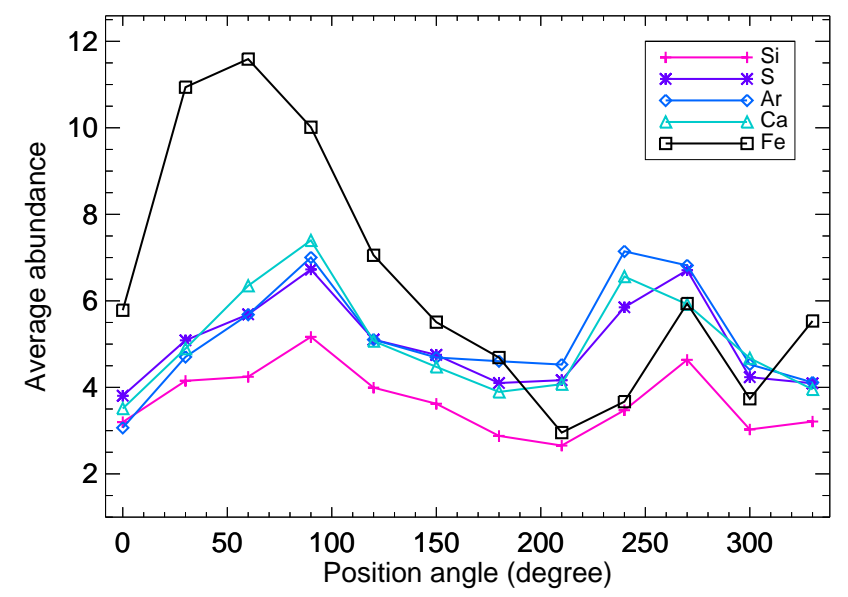

Fig. 10. Average abundances as a function of the P.A. $\left(=0^{\circ}\right.$ to the north); counter-clockwise orientation). The abundance of each element is averaged inside the dashed circle denoted in Fig. 4.

$\alpha_{\mathrm{J} 2000}=19^{\mathrm{h}} 11^{\mathrm{m}} 07^{\mathrm{s}} .6, \delta_{\mathrm{J} 2000}=09^{\circ} 06^{\prime} 10^{\prime} \cdot 2$, which is denoted by a green cross in the bottom-right panel of Fig. 2. In particular, the IGEs, synthesized in the densest part of the exploding WD, show strong lateral distribution. The abundance of $\mathrm{Fe}$ is evidently enhanced in the eastern part of $\mathrm{W} 49 \mathrm{~B}$, and in the range PA $\sim 10-130^{\circ}$ (mean $[\mathrm{Fe}]>6$ ). The $\mathrm{Si}-\mathrm{Ca}$ abundances are elevated in a similar P.A. range, but also in a nearly opposite PA range $\left(\sim 210-300^{\circ}\right)$. The lateral distribution of Fe likely reflects intrinsic asymmetries of the SN explosion. The IMEs have a 
more axial symmetric morphology, which could be intrinsic to the explosion as well, but as discussed in Sect. 3.3 could also be caused by the structure of the circumstellar medium in which $\mathrm{W} 49 \mathrm{~B}$ is evolving.

A feasible explanation for an asymmetric Type-Ia SN explosion is off-center ignition of a WD (Röpke et al. 2007; Maeda et al. 2010b), which has been used to interpret the spectral evolution diversity observed in Type-Ia SNe (Maeda et al. 2010b). Maeda et al. (2010a) modeled nucleosynthesis results of an extremely off-center deflagration of a Chandrasekhar-mass WD followed with a DDT (model "O-DDT"), and compare them with those from a spherically symmetric explosion (model "C-DDT"). As shown in the bottom-right panel of Fig. 9, the off-center ignition model (29 sparks assumed) better describes the abundance ratios in W49B. Furthermore, the off-center ignition model predicts an offset distribution of stable $\mathrm{Fe}$-peak elements, which appears to explain the lateral Fe distribution. The abundances and yields of two-dimensional simulations of an off-center SN explosion are identical to those of the 100 sparks ignition model as shown in the bottom-left panel of Fig. 9 (see Seitenzahl et al. 2013b, for more comparison of the two models), and also similar to the nucleosynthesis results of a recent gravitational detonation model with a single off-center bubble ignition (Seitenzahl et al. 2016).

Although the current study does not pin down a specific model among many Type-Ia models, we have found that W49B may provide a unique and important example of an asymmetric Type-Ia explosion, which may offer more clues about how WDs can explode asymmetrically.

\subsubsection{The high $\mathrm{Mn}$ abundance and its implications}

Most of the ${ }^{55} \mathrm{Mn}$ is produced by the decay of ${ }^{55} \mathrm{Co}$ via ${ }^{55} \mathrm{Fe}$, while the ${ }^{55} \mathrm{Co}$ is mainly synthesized in the incomplete $\mathrm{Si}$ burning and "normal" freeze-out from nuclear statistical equilibrium (NSE; Seitenzahl et al. 2013a). The production of the neutron-rich element ${ }^{55} \mathrm{Co}$ (and hence ${ }^{55} \mathrm{Mn}$ ) requires the presence of neutron-rich elements (Badenes et al. 2008; Bravo 2013). The abundance of these elements are influenced by several effects. First of all, it may be caused by the presence of the initial abundance of the neutron-rich element ${ }^{14} \mathrm{~N}$, in which case a high ${ }^{55} \mathrm{Mn}$ yield may reflect the high initial metallicity of the progenitor (Timmes et al. 2003). Using a one-dimensional TypeIa explosion model (spherical, single ignition), Badenes et al. (2008) proposed a relation $M_{\mathrm{Mn}} / M_{\mathrm{Cr}}=5.3 \times Z^{0.65}$ for estimating the progenitor $Z$ with the $\mathrm{Mn}$ and $\mathrm{Cr}$ supernova yields. Secondly, in accreting $\mathrm{C} / \mathrm{O}$ WDs close to the Chandrasekhar limit, the rise in temperatures in the core will result in carbon fusion several centuries before the onset of the explosion. During this so-called carbon-simmering phase, weak interactions enhance the neutron fraction in the core (Piro \& Bildsten 2008), resulting in a higher ${ }^{55} \mathrm{Mn}$ yield of the ensuing Type-Ia supernova. Finally, a high yield of ${ }^{55} \mathrm{Mn}$ from "normal" freeze-out from NSE requires that the nucleosynthesis occurs under conditions of high density $\left(\rho \gtrsim 2 \times 10^{8} \mathrm{~cm}^{-3}\right.$; Thielemann et al. 1986). Such high density can be provided by WDs with masses larger than $1.2 M_{\odot}$, which is inconsistent with the sub-Chandrasekhar WD scenarios (Seitenzahl et al. 2013a). But the high yield of Mn is consistent with the single-degenerate Type-Ia scenario, for which the mass of the exploding WD should be close to the Chandrasekhar limit. Even for Chandrasekhar-limit explosions, the $\mathrm{Mn} / \mathrm{Fe}$ abundance ratio is rather sensitive to the explosion conditions.

For a few young SNRs, such as Tycho's and Kepler's SNRs, the high $\mathrm{Mn} / \mathrm{Cr}$ ratio has been taken as evidence for a high initial metallicity of the progenitor (Badenes et al. 2008; Park et al. 2013), since in these cases mostly the outer layers of the supernovae were assumed to be shocked, and the ${ }^{55} \mathrm{Mn}$ yield in the outer layer does depend on the initial metallicity. Carbon simmering as the cause of the high Mn yield seems unlikely, since it only affects the neutron excess of the core. However, a high metallicity was suggested in the young SNR N103B based on metal ratios, which seems highly unlikely given that this SNR is located in the low-metallicity environment of the Large Magellanic Cloud (Badenes 2016). In this case, but perhaps also in hindsight for the cases of Tycho's and Kepler's SNRs, the high yields may be affected by simmering, and radial mixing of material from the core and outer regions (e.g., Gamezo et al. 2005) may have affected the ${ }^{55} \mathrm{Mn}$ abundance of outer ejecta (but see Badenes et al. 2005). Finally, a large $\mathrm{Mn} / \mathrm{Cr}$ ratio was also found in the Type-Ia SNR candidate 3C 397, which could be contributed by the neutron-rich NSE region (Yamaguchi et al. 2015). On the other hand, a dense, low-carbon WD with a solarand subsolar-metallicity progenitor can also produce the high $\mathrm{Mn} / \mathrm{Cr}$ ratio in 3C 397 (Dave et al. 2017).

We report here a Mn mass of $M_{\mathrm{Mn}}=6.6 \pm 1.9 \times 10^{-3} M_{\odot}$ for W49B, based on fitting the X-ray spectra with the vrnei model. The mass ratio of $\mathrm{Mn}$ and $\mathrm{Cr}$ is $\sim 1.3(0.8-2.2)$. If we would attribute this to the effect of progenitor metallicity, a super-solar metalicity of $Z=0.12_{-0.07}^{+0.14}=8_{-4}^{+10} Z_{\odot}$ is implied (using the solar ratio of Asplund et al. 2009).

W49B is in a later stage of its SNR evolution than young SNRs like Tycho's and Kepler's SNR, so the $\mathrm{Mn} / \mathrm{Cr}$ ratios reported here likely reflect the combination of both core and outer ejecta, that is, from both "normal" freeze-out from NSE and incomplete silicon burning. In this case, the ratio also depends on the selected initial parameters such as central density and numbers of flaming bubbles (Seitenzahl et al. 2013b) and the explosion mechanisms (e.g., Dave et al. 2017).

As shown in the bottom-left panel of Fig. 9, the threedimensional models with solar-metallicity progenitor and different ignition sparks predict a range of $M_{\mathrm{Mn}} / M_{C r}(0.6-2.2)$, which overlaps with the mass ratio of $1.3(0.8-2.2)$ reported here for W49B. Therefore, from the point of view of existing models, and the SNR phase W49B in super-solar metallicity models may not be required to explain our estimated high $\mathrm{Mn} / \mathrm{Cr}$ mass ratio. Moreover, the high $\mathrm{Mn} / \mathrm{Cr}$ ratio, and the high $\mathrm{Mn} / \mathrm{Fe}$ ratio of $[\mathrm{Mn} / \mathrm{Fe}] /[\mathrm{Mn} / \mathrm{Fe}]_{\odot}$ of $1.4-3.3$ in W49B strongly suggest a WD explosion almost the mass of Chandrasekhar (sub-solar $\mathrm{Mn} / \mathrm{Fe}$ ratio for sub-Chandrasekhar cases; Seitenzahl et al. 2013a), which is best explained by the single-degenerate scenario.

\subsubsection{Properties for typing the SNR}

Table 1 summarizes the main properties for typing an SNR and the results of $\mathrm{W} 49 \mathrm{~B}$, in which their importance in reference is reflected in the sequence.

$P W N / N S$ : There is no PWN detected inside W49B, or any clear evidence of an associated NS, although the presence of an NS cannot yet be excluded, as shown by the presence of numerous point sources in and around W49B (Fig. 6). An association of one of them with an NS would strongly suggest a CC origin.

Ejecta: The masses of the IGEs in the SNR are heavily overabundant compared to $\mathrm{CC}$ nucleosynthesis models. The observed abundance ratios instead suggest a Type-Ia origin.

Asymmetry: CC SNRs appear to be statistically more asymmetrical than Type-Ia SNRs (Lopez et al. 2011). However, there is emerging evidence that a fraction of the Type-Ia SN explosion 
Table 1. Properties for typing SNRs and W49B's results.

\begin{tabular}{lcccc}
\hline \hline Properties & W49B & Bipolar CC & Normal CC & Type-Ia \\
\hline PWN & No & + & \pm & + \\
NS & Unclear & \pm & \pm & \pm \\
Ejecta & Iron-group rich & - & - & + \\
Asymmetry & Yes & + & + & \pm \\
Wind bubble & $\lesssim 6 \mathrm{pc}$ & - & + & \pm \\
\hline
\end{tabular}

Notes. The - and + symbols under each explosion mechanism indicate negative and positive evidence, respectively, given the properties of W49B listed in the second column. The \pm symbols indicate that some known SNRs (not for all) with similar properties match the scenario, or that the explosion mechanism cannot be constrained due to some unclear property.

is intrinsically aspherical (Maeda et al. 2010b; Uchida et al. 2013, for SN 1006). Moreover, the morphology of evolved SNR is subject to the shaping by the inhomogeneous environment. Given the nonuniform environment, Type-Ia SNe can also evolve to mixed-morphology SNRs. For W49B, the centrally filled morphology is mainly due to density enhancement in the SNR interior (see discussion below). We note that also the candidate Type-Ia SNR 3C 397 (Chen et al. 1999; Safi-Harb et al. 2000; Yang et al. 2013; Yamaguchi et al. 2014, 2015, the last reference suggests a CC origin) is highly aspherical, with many properties that are similar to W49B.

Wind bubble: The presence and size of a wind bubble, and/or the stellar environment itself provides clues about the progenitors of SNRs. W49B is suggested to be located inside a wind-blown bubble, whose relatively small size suggests that the progenitor had a mass of $\sim 13 M_{\odot}$, or smaller (if the SNR shock has already transgressed sufficiently into the shell) if it is a CC SNR. However, Type-Ia SNRs can also be associated with wind bubbles if there is outflow driven by their single-degenerate progenitor systems, such as Tycho (Zhou et al. 2016) and the Type-Ia candidate RCW 86 (Badenes et al. 2007; Williams et al. 2011; Broersen et al. 2014). Moreover, observations of Type-Ia SNe have shown that a considerable population explodes "promptly" and associated relatively young stellar populations, although the delay time of their explosions (40-420 Myr) is still larger than that of CC SNe ( $\lesssim 40 \mathrm{Myr}$; Maoz et al. 2012). The presence of a small cavity surrounded by a dense ambient medium therefore either suggests a CC SNR with a low-mass progenitor, or a Type-Ia SNR, with a moderately sized wind-blown bubble.

We favor the Type-Ia origin of W49B after a comparison of all direct and indirect properties for SNR typing, although some of its indirect properties can also be explained by a normal CC explosion. Both observations and models indicate that there is some diversity among Type-Ia SNe. In that regard, W49B is an intriguing object revealing some properties different from other Type-Ia SNRs. Further studies, especially on the details of the explosion process and its environment, are needed to achieve an integrated understanding of this remnant.

\section{3. "Bar-like" morphology due to a density enhancement}

The bar-like X-ray morphology, which has an east-west orientation and is sharper in Fe-K emission, has been attributed to either an asymmetric SN explosion origin (Miceli et al. 2006; Lopez et al. 2013b), the density distribution of the ambient medium (Miceli et al. 2006) shaped by the progenitor winds (Keohane et al. 2007; Miceli et al. 2008; Zhou et al. 2011) or to two opposite SN jets perpendicular to the bar-like structure (Bear \& Soker 2017).
Our density maps show that the cold component reveals a high-density distribution that has a similar east-west orientation, which is less prominently present in the hot component. The strong association with the cold component, which is likely shocked ambient medium, suggests that the bar-like structure is most likely the result of a structure in the ambient medium.

However, the X-ray morphology is clearly a combination of the metal-rich ejecta distribution and the density distribution. A possible explanation is that $\mathrm{W} 49 \mathrm{~B}$ evolves inside a more or less barrel-shaped cavity, with lower densities in the North and South, which agrees well with the shape of the infrared (Keohane et al. 2007) and radio emission (Moffett et al. 1993). The higher densities in the equatorial direction are reflected in the higher densities of the cold component along the bar. The high densities along the bar probably also triggered the early formation of the reverse shock, and therefore also resulted in the ejecta being shocked at higher densities in the central region of W49B.

The intrinsic asymmetry of the ejecta is reflected not in the overall bar-like morphology of the X-ray line emission, but in the abundance maps (Fig. 2), which indeed reveal not so much a bar-like morphology, but a fan-like region that reveals higher $\mathrm{Fe}$ abundances in the Eastern part of the bar-like region.

A bipolar explosion jet seems unlikely, since the base of the jet should be displaced several thousand years after the explosion (see the numerical simulation in González-Casanova et al. 2014). Instead the bar-like region goes through the center of the W49B, which is why this SNR is labeled a mixed-morphology SNR.

\subsection{Recombining gas and SNR age}

As mentioned in Sect. 2.4, the recombining plasma occupies the majority of the SNR regions of W49B ( 3/4 by area), not only in the southwest, but also in the bar-like structure in the SNR interior and in the northeast. The recombination age in the northeast is larger than in the southwest (see Fig. 4). The gas that has reached CIE is distributed in the southeastern shell and spreads in patches throughout the northeastern part of the remnant.

The distribution and origin of the recombining/over-ionized plasma in W49B has been studied in several papers, and rapid cooling is regarded as the cause (e.g., Ozawa et al. 2009). Thermal conduction was initially proposed as the cooling mechanism (Kawasaki et al. 2005), but adiabatic expansion is more favored in the later studies (Miceli et al. 2010; Lopez et al. 2013a). Zhou et al. (2011) performed a hydrodynamic simulation of the W49B evolution in a circumstellar dense cylinder as indicated by the infrared observations. They found that the recombining plasma originates in both cooling processes: (1) the mixing of hot plasma and cold gas evaporated from the dense circumstellar medium; and (2) rapid adiabatic expansion. The density enhancement along the bar-like structure (see Fig. 2) supports the existence of the dense circumstellar matter for process (1) to occur. The adiabatic expansion can explain the large-scale recombining plasma extended to the southwestern boundary.

The recombination age shown in Fig. 4 is derived from the recombination timescale and the electron density in the hot phase $\left(t_{\mathrm{r}}=\tau_{\mathrm{r}} / n_{e}\right.$, where the density is assumed to be a constant with time). It describes the time elapse starting from the moment that plasma reached ionization equilibrium, and then began cooling. Our measurements indicate recombination ages between $\sim 2000$ and $\sim 6000 \mathrm{yr}$, based on an over-ionization model. The true age of the plasma may well differ as the ionization state depends on the temperature and density history of the plasma. But one should also note that over-ionization can only occur once ionization equilibrium has been reached, and the plasma turns 
from under-ionized into over-ionized (Kawasaki et al. 2005). The latter would imply that our recombination age measurements even underestimate the true plasma age. Although the connection between recombination age and SNR age is complex, the recombination ages that we find have implications for the age of W49B.

In previous studies, the age of W49B was estimated to be between $1000 \mathrm{yr}$ (Pye et al. 1984; Lopez et al. 2013b) and $\sim 4000 \mathrm{yr}$ (Hwang et al. 2000). The recombination ages measured by us suggest that the SNR is older, as the SNR cannot be younger than the oldest plasma it contains, and the recombination age probably underestimates the true age of the plasma. Moreover, for the plasma to first reach ionization equilibrium, a timescale of $\gtrsim 10^{12} \mathrm{~cm}^{-3} \mathrm{~s}$ (Smith \& Hughes 2010) is needed. Even for densities around $n_{\mathrm{H}} \approx 10 \mathrm{~cm}^{-3}$, typical for the Western region, the associated timescale is $\gtrsim 2600 \mathrm{yr}$. The recombination timescale of $\sim 2000 \mathrm{yr}$ for that region should be added to that. Therefore, the total plasma age must be of the order of 4000-5000 yr. In the eastern regions, the recombination ages are as high as $6000 \mathrm{yr}$, or the plasma is in equilibrium (white pixels in Fig. 4). Since the density is lower in that region $\left(n_{\mathrm{H}} \approx 4 \mathrm{~cm}^{-3}\right)$, CIE implies plasma ages of $\sim 6600 \mathrm{yr}$, close to maximum recombination ages we find. The recombination ages therefore suggest an age of at least 5000-6000 yr.

One can also estimate the age based on the Sedov model combined with an estimate of the forward shock velocity based on the plasma temperature. Here one has the choice between taking the hot plasma component or the cool plasma component. However, the hot plasma component seems to be very much metal enriched, and shows a lot of temperature variation. This component is likely associated with plasma shocked by the reverse shock. Associating the cooler component with the forward shock, we infer a forward shock velocity of $v_{\mathrm{s}}=$ $\left[16 k T_{\mathrm{c}} /\left(3 \bar{\mu} m_{\mathrm{H}}\right)\right]^{1 / 2}=476\left(k T_{\mathrm{c}} / 0.27 \mathrm{keV}\right)^{1 / 2} \mathrm{~km} \mathrm{~s}^{-1}$, where the mean atomic weight $\bar{\mu}=0.61$ is taken for fully ionized plasma and $m_{\mathrm{H}}$ is the hydrogen atomic mass. For a uniform ambient medium, the velocity corresponds to a Sedov age of $t_{\text {sedov }}=$ $2 R_{\mathrm{s}} /\left(5 v_{\mathrm{s}}\right) \sim 5.3\left(k T_{\mathrm{c}} / 0.27 \mathrm{keV}\right)^{-1 / 2} \mathrm{kyr}$, and an explosion energy $E_{0}=25 /(4 \xi)\left(1.4 n_{0} m_{\mathrm{H}}\right) R_{\mathrm{s}}^{3} v_{\mathrm{s}}^{2} \sim 1.3 \times 10^{51} n_{10}$ erg (Ostriker \& McKee 1988), where we adopt an SNR radius $R_{\mathrm{S}}=6.4 \mathrm{pc}$, and $\xi=2.026$, with $n_{10}$ the ambient density of hydrogen atom in units of $10 \mathrm{~cm}^{-3}$. The temperature of the cool component is therefore also consistent with an age of 5000-6000 yr, and the canonical supernova explosion energy of $\sim 10^{51} \mathrm{erg}$ for the high density environment in which W49B expands.

\section{Conclusion}

We have performed a spatially resolved X-ray study of SNR W49B using a state-of-the-art adaptive binning method in order to uncover its explosion mechanism and the origin of the centrally filled morphology. An asymmetric Type-Ia explosion is the most probable explanation for the abundances, yields, and metal distribution in W49B. A density enhancement with an east-west orientation is the main reason for the bar-like X-ray morphology. The detailed results are summarized as follows.

- The X-ray emission in W49B is well characterized by two-temperature gas containing a cool component with $k T_{\mathrm{c}} \sim 0.27 \mathrm{keV}$ and a hot, ejecta-rich component with $k T_{\mathrm{h}} \sim 0.6-2.2 \mathrm{keV}$. There is a large gradient of $k T_{\mathrm{h}}$ from the northeast to the southwest. The detailed distribution of gas temperature and other physical parameters across the SNR are shown (see Figs. 2 and 4).
- W49B is evolving in a dense environment. The mean densities are 24 and $5 \mathrm{~cm}^{-3}$ for the cool and hot X-ray components, respectively. The total masses of the X-rayemitting gas are $484_{-34}^{+41} d_{10}^{2.5} M_{\odot}$ in the cool phase and $52 \pm 8 d_{10}^{2.5} M_{\odot}$ in the hot phase.

- We obtained the mass-weighted average abundances $[\mathrm{Si}]=3.4_{-0.7}^{+0.8},[\mathrm{~S}]=4.9_{-1.0}^{+1.1},[\mathrm{Ar}]=5.1_{-1.0}^{+1.2},[\mathrm{Ca}]=5.2_{-1.0}^{+1.2}$, and $[\mathrm{Fe}]=5.7_{-1.1}^{+1.2}$. The $\mathrm{Cr}, \mathrm{Mn}$, and $\mathrm{Fe}$ abundances according to a fit to the global spectra of $\mathrm{W} 49 \mathrm{~B}$ are $[\mathrm{Cr}]=6.6 \pm 0.8$, $[\mathrm{Mn}]=12.5 \pm 2.7$, and $[\mathrm{Fe}]=3.2 \pm 0.1$.

- The element Fe shows a strong lateral distribution. The abundance of $\mathrm{Fe}$ is evidently enhanced in $\mathrm{PA}$ range $\sim 10-130^{\circ}$ (SNR east). The $\mathrm{Si}-\mathrm{Ca}$ abundances are also elevated in a similar PA range, but also in the nearly opposite P.A. range $\left(\sim 210-300^{\circ}\right)$. The lateral distribution of Fe suggests intrinsic asymmetries of the SN explosion. The nearly axially symmetric distribution of IMEs may also reflect that the explosion was not sphericially symmetric, but in this case also the density distribution of the circumstellar medium may play a role (see point 8 below).

- We have found 24 point-like sources in the vicinity of W49B with luminosities in the range $10^{32.1-36.2} d_{10}^{2} \mathrm{erg} \mathrm{s}^{-1}$. Nine of them have the luminosities of a cooling NS at the age of W49B (at an assumed distance of $10 \mathrm{kpc}$ ). Therefore, even if $\mathrm{W} 49 \mathrm{~B}$ is a CC SNR, there is still the possibility that it contains a cooling NS, rather than a black hole.

- None of the CC nucleosynthesis models (spherical explosion or bipolar explosion) explain the abundance ratios in W49B. The iron-group yields predicted by the $\mathrm{CC}$ models are insufficient to explain the observed masses in the hotter phase: $M_{\mathrm{Cr}}=4.9 \pm 1.1 \times 10^{-3} M_{\odot}, M_{\mathrm{Mn}}=6.6 \pm 1.9 \times 10^{-3} M_{\odot}$, $M_{\mathrm{Fe}}=0.32_{-0.09}^{+0.10} M_{\odot}$. The energetic CC explosion scenario matches even more poorly than the normal $\mathrm{CC}$ scenario given the small molecular cavity surrounding the SNR and nine point-like sources in the vicinity of W49B (probably only a projection effect).

- A DDT Type-Ia model with multi-spot ignition of a Chandrasakhar-mass WD well describes the observed abundance ratios. This model based on solar-metallicity can also explain the high $\mathrm{Mn}$-to-Cr ratio $\left(M_{\mathrm{Mn}} / M_{\mathrm{Cr}}=0.8-2.2\right)$ found in W49B. A feasible explanation of the asymmetric Type-Ia $\mathrm{SN}$ explosion is off-center ignition of a WD.

- The centrally filled/bar-like morphology of W49B is mainly due to density enhancement projected to the SNR center $\left(n_{c}>30 \mathrm{~cm}^{-3}\right)$, given the good spatial correlation between the gas density and the X-ray brightness. This suggests that W49B evolves in a barrel-shaped cavity, which also lead the ejecta to be shocked at higher densities and projected to the center. The overall morphology, triggered by the ambient medium structure, combined with our conclusion that W49B is a Type-Ia SNR, suggests that Type-Ia supernovae can also result in mixed-morphology SNRs.

- The recombination age of the plasma suggests an SNR age of 5-6 kyr, similar to the estimated Sedov age of $5.3 \mathrm{kyr}$.

Acknowledgements. P.Z. acknowledges the support from the NWO Veni Fellowship, grant no. 639.041.647 and NSFC grants 11503008, 11590781, and 11233001 .

\section{References}

H. E. S. S. Collaboration (Abdalla, H., et al.) 2018, A\&A, 612, A5 Akmal, A., Pandharipande, V. R., \& Ravenhall, D. G. 1998, Phys. Rev. C, 58, 1804 
Anders, E., \& Grevesse, N. 1989, Geochim. Cosmochim. Acta, 53, 197

Asplund, M., Grevesse, N., Sauval, A. J., \& Scott, P. 2009, ARA\&A, 47, 481

Badenes, C. 2016, Supernova Remnants: An Odyssey in Space After Stellar Death, 105

Badenes, C., Borkowski, K. J., \& Bravo, E. 2005, ApJ, 624, 198

Badenes, C., Hughes, J. P., Bravo, E., \& Langer, N. 2007, ApJ, 662, 472

Badenes, C., Bravo, E., \& Hughes, J. P. 2008, ApJ, 680, L33

Bear, E., \& Soker, N. 2017, MNRAS, 468, 140

Blitz, L. 1993, Protostars and Planets III, eds. E. H. Levy, \& J. I. Lunine (Tucson: Univ. of. Arizona), 125

Borkowski, K. J., Rho, J., Reynolds, S. P., \& Dyer, K. K. 2001, ApJ, 550, 334

Branch, D., Livio, M., Yungelson, L. R., Boffi, F. R., \& Baron, E. 1995, PASP, 107, 1019

Bravo, E. 2013, A\&A, 550, A24

Broersen, S., Chiotellis, A., Vink, J., \& Bamba, A. 2014, MNRAS, 441, 3040

Brogan, C. L., \& Troland, T. H. 2001, ApJ, 550, 799

Cappellari, M., \& Copin, Y. 2003, MNRAS, 342, 345

Chen, Y., Sun, M., Wang, Z.-R., \& Yin, Q. F. 1999, ApJ, 520, 737

Chen, Y., Zhou, P., \& Chu, Y.-H. 2013, ApJ, 769, L16

Chen, Y., Jiang, B., Zhou, P., et al. 2014, in Supernova Environmental Impacts, eds. A. Ray, \& R. A. McCray, IAU Symp., 296, 170

Chevalier, R. A. 1999, ApJ, 511, 798

Chiotellis, A., Schure, K. M., \& Vink, J. 2012, A\&A, 537, A139

Dave, P., Kashyap, R., Fisher, R., et al. 2017, ApJ, 841, 58

Diehl, S., \& Statler, T. S. 2006, MNRAS, 368, 497

Dubner, G., \& Giacani, E. 2015, A\&ARv, 23, 3

Gamezo, V. N., Khokhlov, A. M., \& Oran, E. S. 2005, ApJ, 623, 337

Garcia-Senz, D., \& Woosley, S. E. 1995, ApJ, 454, 895

González-Casanova, D. F., De Colle, F., Ramirez-Ruiz, E., \& Lopez, L. A. 2014, ApJ, 781, L26

Gvaramadze, V. V., Langer, N., Fossati, L., et al. 2017, Nat. Astron., 1, 0116

Heger, A., Fryer, C. L., Woosley, S. E., Langer, N., \& Hartmann, D. H. 2003, ApJ, 591, 288

Helfand, D. J., Becker, R. H., White, R. L., Fallon, A., \& Tuttle, S. 2006, AJ, 131, 2525

Hillebrandt, W., \& Niemeyer, J. C. 2000, ARA\&A, 38, 191

Hughes, J. P., Hayashi, I., \& Helfand, D., et al. 1995, ApJ, 444, L81

Hwang, U., Petre, R., \& Hughes, J. P. 2000, ApJ, 532, 970

Iapichino, L., Brüggen, M., Hillebrandt, W., \& Niemeyer, J. C. 2006, A\&A, 450, 655

Jones, T. W., Rudnick, L., Jun, B.-I., et al. 1998, PASP, 110, 125

Kawasaki, M., Ozaki, M., Nagase, F., Inoue, H., \& Petre, R. 2005, ApJ, 631, 935

Keohane, J. W., Reach, W. T., Rho, J., \& Jarrett, T. H. 2007, ApJ, 654, 938

Khokhlov, A. M. 1991, A\&A, 245, L25

Kinugasa, K., \& Tsunemi, H. 1999, PASJ, 51, 239

Lazendic, J. S., \& Slane, P. O. 2006, ApJ, 647, 350

Livio, M. 2000, eds. J. C. Niemeyer, \& J. W. Truran, Type Ia Supernovae, Theory and Cosmology (Cambridge: Cambridge University Press), 33

Lopez, L. A., Ramirez-Ruiz, E., Huppenkothen, D., Badenes, C., \& Pooley, D. A. 2011, ApJ, 732, 114

Lopez, L. A., Pearson, S., Ramirez-Ruiz, E., et al. 2013a, ApJ, 777, 145

Lopez, L. A., Ramirez-Ruiz, E., Castro, D., \& Pearson, S. 2013b, ApJ, 764, 50

Maeda, K., \& Nomoto, K. 2003, ApJ, 598, 1163

Maeda, Y., Baganoff, F. K., Feigelson, E. D., et al. 2002, ApJ, 570, 671

Maeda, K., Benetti, S., Stritzinger, M., et al. 2010a, Nature, 466, 82

Maeda, K., Röpke, F. K., Fink, M., et al. 2010b, ApJ, 712, 624

Maoz, D., Mannucci, F., \& Brandt, T. D. 2012, MNRAS, 426, 3282

Maoz, D., Mannucci, F., \& Nelemans, G. 2014, ARA\&A, 52, 107

Miceli, M., Decourchelle, A., Ballet, J., et al. 2006, A\&A, 453, 567

Miceli, M., Decourchelle, A., Ballet, J., et al. 2008, Adv. Space Res., 41, 390

Miceli, M., Bocchino, F., Decourchelle, A., Ballet, J., \& Reale, F. 2010, A\&A, 514, L2

Moffett, D. A., Goss, W. M., \& Reynolds, S. P. 1993, AJ, 106, 1566
Nomoto, K., Thielemann, F.-K., \& Yokoi, K. 1984, ApJ, 286, 644

Nomoto, K., Tominaga, N., Umeda, H., Kobayashi, C., \& Maeda, K. 2006, Nucl. Phys. A, 777, 424

Olbert, C. M., Clearfield, C. R., Williams, N. E., Keohane, J. W., \& Frail, D. A. 2001, ApJ, 554, L205

Ostriker, J. P., \& McKee, C. F. 1988, Rev. Mod. Phys., 60, 1

Ozawa, M., Koyama, K., Yamaguchi, H., Masai, K., \& Tamagawa, T. 2009, ApJ, 706, L71

Page, D., Lattimer, J. M., Prakash, M., \& Steiner, A. W. 2004, ApJS, 155, 623 Page, D., Lattimer, J. M., Prakash, M., \& Steiner, A. W. 2009, ApJ, 707, 1131

Park, S., Muno, M. P., Baganoff, F. K., et al. 2005, ApJ, 631, 964

Park, S., Slane, P. O., Hughes, J. P., et al. 2007, ApJ, 665, 1173

Park, S., Badenes, C., Mori, K., et al. 2013, ApJ, 767, L10

Pavlov, G. G., Sanwal, D., \& Teter, M. A. 2004, in Young Neutron Stars and Their Environments, eds. F. Camilo, \& B. M. Gaensler, IAU Symp., 218, 239 Piro, A. L., \& Bildsten, L. 2008, ApJ, 673, 1009

Post, S., Park, S., Badenes, C., et al. 2014, ApJ, 792, L20

Pye, J. P., Becker, R. H., Seward, F. D., \& Thomas, N. 1984, MNRAS, 207, 649

Radhakrishnan, V., Goss, W. M., Murray, J. D., \& Brooks, J. W. 1972, ApJS, 24, 49

Reynolds, S. P., Lyutikov, M., Blandford, R. D., \& Seward, F. D. 1994, MNRAS, 271, L1

Rho, J., \& Petre, R. 1998, ApJ, 503, L167

Röpke, F. K., Hillebrandt, W., Schmidt, W., et al. 2007, ApJ, 668, 1132

Safi-Harb, S., Petre, R., Arnaud, K. A., et al. 2000, ApJ, 545, 922

Sakano, M., Warwick, R. S., Decourchelle, A., \& Predehl, P. 2004, MNRAS, 350,129

Seitenzahl, I. R., \& Townsley, D. M. 2017, ArXiv e-prints [arXiv:1704.00415]

Seitenzahl, I. R., Cescutti, G., Röpke, F. K., Ruiter, A. J., \& Pakmor, R. 2013a, A\&A, 559, L5

Seitenzahl, I. R., Ciaraldi-Schoolmann, F., Röpke, F. K., et al. 2013b, MNRAS, 429, 1156

Seitenzahl, I. R., Kromer, M., Ohlmann, S. T., et al. 2016, A\&A, 592, A57

Smith, R. K., \& Hughes, J. P. 2010, ApJ, 718, 583

Smith, A., Peacock, A., Jones, L. R., \& Pye, J. P. 1985, ApJ, 296, 469

Sukhbold, T., Ertl, T., Woosley, S. E., Brown, J. M., \& Janka, H.-T. 2016, ApJ, 821,38

Tananbaum, H. 1999, IAU Circ., 7246, 1

Thielemann, F.-K., Nomoto, K., \& Yokoi, K. 1986, A\&A, 158, 17

Timmes, F. X., Brown, E. F., \& Truran, J. W. 2003, ApJ, 590, L83

Uchida, H., Yamaguchi, H., \& Koyama, K. 2013, ApJ, 771, 56

Vink, J. 2012, A\&ARv, 20, 49

Vink, J. 2016, Supernova 1604, Kepler's Supernova, and its Remnant (Cham: Springer), Handbook of Supernovae [arXiv:1612.06905]

Wang, B., \& Han, Z. 2012, New Astron. Rev., 56, 122

Weaver, R., McCray, R., Castor, J., Shapiro, P., \& Moore, R. 1977, ApJ, 218, 377

Webbink, R. F. 1984, ApJ, 277, 355

Whelan, J., \& Iben, Jr., I. 1973, ApJ, 186, 1007

Williams, B. J., Blair, W. P., Blondin, J. M., et al. 2011, ApJ, 741, 96

Wilms, J., Allen, A., \& McCray, R. 2000, ApJ, 542, 914

Winkler, P. F., Gupta, G., \& Long, K. S. 2003, ApJ, 585, 324

Wolszczan, A., Cordes, J. M., \& Dewey, R. J. 1991, ApJ, 372, L99

Woosley, S. E., Wunsch, S., \& Kuhlen, M. 2004, ApJ, 607, 921

Yamaguchi, H., Ozawa, M., Koyama, K., et al. 2009, ApJ, 705, L6

Yamaguchi, H., Badenes, C., Petre, R., et al. 2014, ApJ, 785, L27

Yamaguchi, H., Badenes, C., Foster, A. R., et al. 2015, ApJ, 801, L31

Yang, X. J., Tsunemi, H., Lu, F. J., et al. 2013, ApJ, 766, 44

Zhang, G.-Y., Chen, Y., Su, Y., et al. 2015, ApJ, 799, 103

Zhou, X., Miceli, M., Bocchino, F., Orlando, S., \& Chen, Y. 2011, MNRAS, 415, 244

Zhou, P., Chen, Y., Zhang, Z.-Y., et al. 2016, ApJ, 826, 34

Zhu, H., Tian, W. W., \& Zuo, P. 2014, ApJ, 793, 95 


\section{Appendix A: Point-like sources in the vicinity of W49B}

Table A.1. Information of the point-like sources in the vicinity of W49B.

\begin{tabular}{|c|c|c|c|c|c|c|c|c|c|c|c|}
\hline \multirow[t]{2}{*}{ No. } & \multicolumn{2}{|c|}{ Coordinates } & \multicolumn{2}{|c|}{ Counts (hard) } & \multicolumn{2}{|c|}{ Counts (soft) } & \multirow{2}{*}{$\begin{array}{l}\text { Significance } \\
(\sigma)\end{array}$} & \multirow{2}{*}{$\begin{array}{c}\text { Photon flux } f \\
\text { (photon } \mathrm{s}^{-1} \mathrm{~cm}^{-2} \text { ) }\end{array}$} & \multirow{2}{*}{$\begin{array}{l}\text { Hardness ratio } \\
\qquad \frac{\left(f_{\mathrm{H}}-f_{\mathrm{S}}\right)}{\left(f_{\mathrm{H}}+f_{\mathrm{S}}\right)} \\
\end{array}$} & \multirow{2}{*}{$\begin{array}{l}k T_{\mathrm{BB}} \\
(\mathrm{keV}) \\
\end{array}$} & \multirow{2}{*}{$\begin{array}{r}\log _{10}\left(L_{\mathrm{BB}}\right) \\
\left(\mathrm{erg} \mathrm{s}^{-1}\right) \\
\end{array}$} \\
\hline & RA & Dec & Total & Net & Total & Net & & & & & \\
\hline 1 & $19: 11: 05.7$ & 09:02:33.6 & 75 & 62.5 & 191 & 163.5 & 29.3 & $1.8 \mathrm{E}-06 \pm 5.8 \mathrm{E}-08$ & $-0.24 \pm 0.02$ & 0.25 & 34.2 \\
\hline 2 & $19: 11: 14.6$ & 09:03:28.5 & 17 & 0.9 & 126 & 73.8 & 11.0 & $4.5 \mathrm{E}-07 \pm 3.9 \mathrm{E}-08$ & $-0.96 \pm 0.08$ & 0.12 & 36.2 \\
\hline 3 & $19: 11: 21.2$ & 09:05:02.7 & 26 & 12.9 & 226 & 168.8 & 17.8 & $1.2 \mathrm{E}-06 \pm 4.6 \mathrm{E}-08$ & $-0.79 \pm 0.03$ & 0.17 & 35.2 \\
\hline 4 & 19:11:06.1 & 09:05:33.4 & 166 & 75.4 & 90 & 6.9 & 5.4 & $7.4 \mathrm{E}-07 \pm 5.1 \mathrm{E}-08$ & $0.89 \pm 0.05$ & 1.18 & 32.4 \\
\hline 5 & 19:10:57.1 & 09:05:39.5 & 35 & 23.7 & 22 & -0.4 & 4.8 & $2.2 \mathrm{E}-07 \pm 3.5 \mathrm{E}-08$ & $1.00 \pm 0.11$ & $>5$ & - \\
\hline 6 & $19: 10: 50.5$ & 09:07:24.7 & 29 & 22.2 & 24 & 13.3 & 9.1 & $6.8 \mathrm{E}-07 \pm 8.3 \mathrm{E}-08$ & $0.44 \pm 0.08$ & 0.42 & 32.8 \\
\hline 7 & $19: 11: 04.0$ & 09:07:37.6 & 155 & 20.6 & 338 & 116.4 & 9.1 & $1.0 \mathrm{E}-06 \pm 6.7 \mathrm{E}-08$ & $-0.54 \pm 0.06$ & 0.21 & 34.4 \\
\hline 8 & $19: 10: 57.4$ & 09:04:02.1 & 1 & -1.0 & 35 & 24.5 & 5.8 & $2.2 \mathrm{E}-07 \pm 3.6 \mathrm{E}-08$ & $-1.00 \pm 0.14$ & $<0.01$ & - \\
\hline 9 & 19:11:04.2 & 09:04:04.0 & 21 & 1.0 & 57 & 22.9 & 6.2 & $1.4 \mathrm{E}-07 \pm 3.6 \mathrm{E}-08$ & $-0.87 \pm 0.27$ & 0.15 & 34.7 \\
\hline 10 & $19: 11: 10.6$ & 09:06:37.3 & 491 & 69.3 & 244 & 51.2 & 5.2 & $9.9 \mathrm{E}-07 \pm 7.0 \mathrm{E}-08$ & $0.38 \pm 0.04$ & 0.40 & 33.1 \\
\hline 11 & $19: 11: 21.8$ & 09:06:43.0 & 9 & 5.2 & 12 & 4.5 & 3.7 & $7.8 \mathrm{E}-08 \pm 2.8 \mathrm{E}-08$ & $0.30 \pm 0.24$ & 0.37 & 32.1 \\
\hline 12 & $19: 11: 07.5$ & 09:06:54.9 & 113 & 21.5 & 87 & -4.4 & 3.3 & $2.1 \mathrm{E}-07 \pm 4.9 \mathrm{E}-08$ & $1.00 \pm 0.17$ & $>5$ & - \\
\hline 13 & 19:11:08.1 & 09:07:27.0 & 90 & 36.1 & 63 & 10.4 & 3.9 & $4.3 \mathrm{E}-07 \pm 4.9 \mathrm{E}-08$ & $0.71 \pm 0.07$ & 0.60 & 32.3 \\
\hline 14 & $19: 11: 09.9$ & 09:09:04.4 & 31 & 10.1 & 77 & 53.3 & 9.3 & $4.1 \mathrm{E}-07 \pm 4.1 \mathrm{E}-08$ & $-0.52 \pm 0.09$ & 0.21 & 34.0 \\
\hline 15 & $19: 11: 05.3$ & 09:02:53.2 & 1 & 0.1 & 7 & 5.3 & 2.3 & $5.6 \mathrm{E}-08 \pm 3.3 \mathrm{E}-08$ & $-0.95 \pm 0.58$ & 0.13 & 34.9 \\
\hline 16 & 19:11:14.1 & 09:03:08.9 & 31 & 21.0 & 22 & 1.2 & 5.5 & $2.1 \mathrm{E}-07 \pm 3.5 \mathrm{E}-08$ & $0.93 \pm 0.12$ & 1.83 & 32.1 \\
\hline 17 & $19: 11: 18.9$ & 09:04:03.8 & 10 & -2.0 & 54 & 23.6 & 5.1 & $1.5 \mathrm{E}-07 \pm 3.4 \mathrm{E}-08$ & $-1.00 \pm 0.23$ & $<0.01$ & - \\
\hline 18 & $19: 11: 12.5$ & 09:05:32.4 & 298 & 27.5 & 400 & 78.6 & 5.0 & $7.1 \mathrm{E}-07 \pm 6.5 \mathrm{E}-08$ & $-0.27 \pm 0.07$ & 0.25 & 33.8 \\
\hline 19 & $19: 10: 59.2$ & 09:05:48.6 & 180 & 51.1 & 136 & 24.7 & 5.2 & $6.2 \mathrm{E}-07 \pm 5.4 \mathrm{E}-08$ & $0.54 \pm 0.05$ & 0.47 & 32.7 \\
\hline 20 & $19: 11: 13.0$ & 09:06:09.3 & 307 & 15.3 & 342 & 60.7 & 4.7 & $5.0 \mathrm{E}-07 \pm 6.5 \mathrm{E}-08$ & $-0.41 \pm 0.12$ & 0.23 & 33.9 \\
\hline 21 & 19:11:10.1 & 09:06:56.0 & 197 & 12.2 & 196 & 53.0 & 4.5 & $4.9 \mathrm{E}-07 \pm 6.6 \mathrm{E}-08$ & $-0.45 \pm 0.13$ & 0.22 & 34.0 \\
\hline 22 & $19: 11: 05.9$ & 09:07:30.9 & 245 & 90.0 & 221 & 52.7 & 8.5 & $1.4 \mathrm{E}-06 \pm 7.0 \mathrm{E}-08$ & $0.48 \pm 0.03$ & 0.44 & 33.1 \\
\hline 23 & 19:11:07.9 & 09:10:19.7 & 1 & -0.7 & 19 & 13.0 & 3.6 & $9.7 \mathrm{E}-08 \pm 2.8 \mathrm{E}-08$ & $-1.00 \pm 0.25$ & $<0.01$ & - \\
\hline 24 & $19: 11: 15.7$ & 09:10:26.3 & 4 & 1.9 & 11 & 7.9 & 3.5 & $8.6 \mathrm{E}-08 \pm 3.3 \mathrm{E}-08$ & $-0.43 \pm 0.32$ & 0.22 & 33.2 \\
\hline
\end{tabular}

We detected 24 point-like sources in the $0.7-5 \mathrm{keV}$ energy band in the vicinity of W49B using the Mexican-Hat wavelet source detection tool wavdetect in CIAO (see Fig. 6). We combined the three Chandra observations to detect faint sources, where the exposure-weighted PSF map was use to run wavdetect on the merged dataset ${ }^{8}$. The energy band is selected to highlight the emission of the point-like sources over the SNR background. The compact object is assumed to have an effective temperature of $0.07-0.5 \mathrm{keV}$ for heavy element atmosphere or $0.11-0.5 \mathrm{keV}$ for Hydrogen atmosphere, with a foreground absorption column density $5.5-8.8 \times 10^{22} \mathrm{~cm}^{-2}$. Two criteria are considered for the NS temperature range: (1) The observed central compact objects show blackbody temperatures in the range $0.2-0.5 \mathrm{keV}$ (Pavlov et al. 2004) and (2) the minimal paradigm (Page et al. 2004) predicts a temperature of $0.11 \mathrm{keV}$ for the light elements envelop and $0.07 \mathrm{keV}$ for the heavy envelop of an NS with an age of $6000 \mathrm{yr}$.

To minimize the contamination from the SNR structures, we extracted the most compact sources using the detection spatial scales of $1, \sqrt{2}$, and 2 pixels. Adding a larger spatial scale of $2 \sqrt{2}$ would result in a detection of 76 sources, while most of these sources are likely clumpy plasma inside the SNR. We examined the detected sources in the hard $(2.5-5 \mathrm{keV})$ and soft $(0.7-2.5 \mathrm{keV})$ bands in order to study their spectral properties. Table A.1 summarizes the coordinates, total (backgroundincluded) and net (background-subtracted) counts in the hard and soft bands, photon fluxes $f$, and hardness ratios $H R$ of the sources, where $f$ is the net count rate divided by the effective area of the camera. The hardness ratio defined as $H R=$ $\left(f_{\mathrm{H}}-f_{\mathrm{S}}\right) /\left(f_{\mathrm{H}}+f_{\mathrm{S}}\right)$ has a value between -1 and 1 , where the $f_{\mathrm{H}}$ and $f_{\mathrm{S}}$ are the photon fluxes in the hard and soft bands, respectively.

The blackbody temperatures $k T_{\mathrm{BB}}$ and luminosities $L_{\mathrm{BB}}$ of the point-like sources are estimated using hardness ratio $H R$ and photon flux $f$ (see Fig. 7). The foreground absorption and distance are assumed to be $N_{\mathrm{H}}=8 \times 10^{22} \mathrm{~cm}^{-2}$ and $10 \mathrm{kpc}$, respectively, the same as those of $\mathrm{W} 49 \mathrm{~B}$. The best-fit $k T_{\mathrm{BB}}$ and $L_{\mathrm{BB}}$ are also listed in Table A.1. 